\title{
Álvaro Pentagna
}

Os aspectos do sono nos pacientes com epilepsia

farmacorresistente e seu impacto sobre a qualidade de vida e o funcionamento social

Dissertação apresentada à Faculdade de Medicina da Universidade de São Paulo para obtenção do título de Mestre em Ciências

Programa de Psiquiatria

Orientadora: $\operatorname{Prof}^{\mathrm{a}}{ }^{\mathrm{Dr}}{ }^{\mathrm{a}}$ Kette Dualibi Ramos Valente

São Paulo

2018 


\section{Dados Internacionais de Catalogação na Publicação (CIP)}

Preparada pela Biblioteca da

Faculdade de Medicina da Universidade de São Paulo

Creprodução autorizada pelo autor

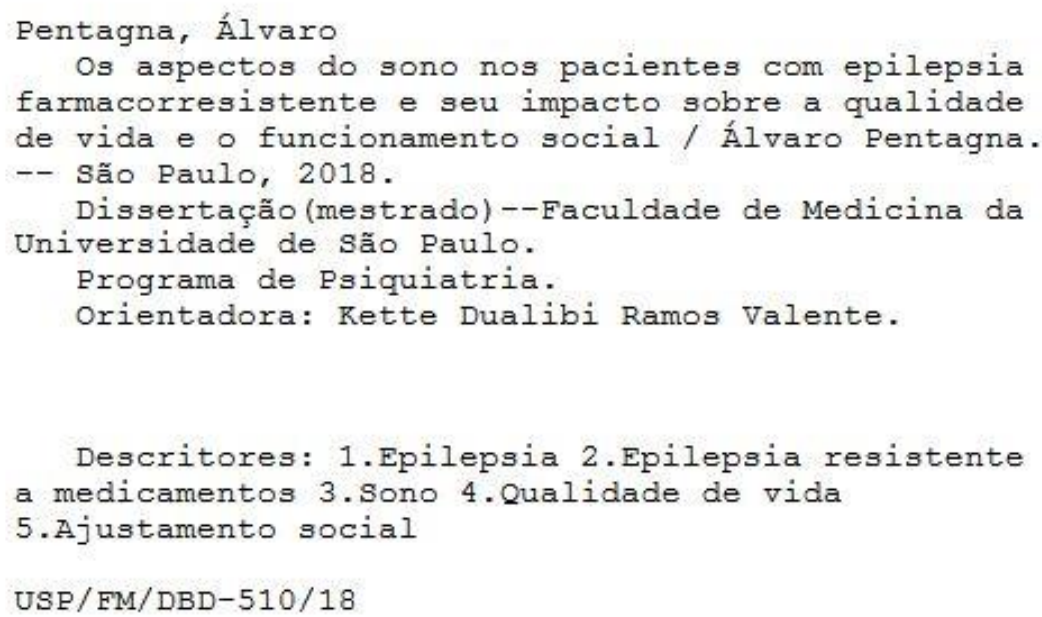

Responsável: Erinalva da Conceição Batista, CRB-8 6755 
A meus avós

Maria José e José Roberto,

Zaïra e Arnoldo. 


\section{AGRADECIMENTOS}

À minha esposa Tatiana, que tanto me apoiou nos momentos de dúvida, decepção e preocupação que ocorrem durante um projeto de pósgraduação.

Aos meus filhos Helena e Paulo, que foram obrigados a ceder momentos de brincadeira com o pai e mesmo assim o amam tanto.

A meus pais e irmãos, que ajudaram a construir a pessoa que sou.

A meus mestres na medicina:

Meus pais, Dr ${ }^{\mathrm{a}}$ Maria Catarina e Dr. Ricardo Pentagna, que me inspiraram na escolha da vocação;

Dr. José Flávio Casteluccio, que na residência de clínica médica na Santa Casa de São Paulo ensinou-me a amar a profissão acima de qualquer adversidade;

Prof. Dr. Paulo Eurípedes Marchiori, que na residência de neurologia no HCFMUSP ensinou-me a estudar de forma incessante;

Drª Stella Márcia Azevedo Tavares e o saudoso Dr. Flávio Sadurny de Alóe que me abriram as portas da medicina do sono;

Dr. Pedro Paulo Porto Júnior, que durante quase dez anos trabalhando juntos no Hospital Israelita Albert Einstein ensinou-me a entender o que os pacientes realmente precisam; 
Prof $^{-}{ }^{-} r^{a}$ Kette Dualibi Ramos Valente, que me guia nos caminhos da pesquisa clínica.

Aos amigos de graduação e clínica médica na Santa Casa de São Paulo, de neurologia no HCFMUSP e de neurofisiologia no Laboratório de Neurofisiologia Clínica do IPq. Todos me acompanharam ou acompanham de algum modo.

À Prof ${ }^{\underline{a}}$ Dra ${ }^{\mathrm{a}}$ Sílvia de Vincentiis, sem a qual a realização deste projeto não teria sido possível.

Ao time de neurologistas do Hospital e Maternidade Rede D'Or São Luiz - Unidade Itaim, que várias vezes me substituíram para que pudesse conduzir a pesquisa.

Às secretárias do IPq, ao Escritório de Apoio à Pesquisa do IPq e à Comissão de Ética do HCFMUSP. Muito me ajudaram a transitar pelas intermináveis burocracias do processo de pesquisa.

Ao estatístico Bernardo dos Santos pela sua paciência com tantas dúvidas que a estatística pode gerar na mente de um médico.

Ao Conselho Nacional de Desenvolvimento Científico e Tecnológico (CNPq), que apoiou a realização deste estudo.

E principalmente a todos os pacientes que participaram da minha formação médica e àqueles que tiveram a paciência e o carinho de participar deste projeto. 
"Oíd, caballeros, ahora, voy a hablaros de verdad:

El que no cambia de sitio perder puede, no ganar."

Trecho do cantar 54 do épico castelhano

Cantar de Mio Cid, séc. XIII d.C. 
Sumário

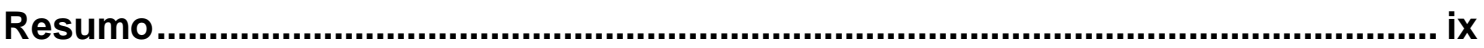

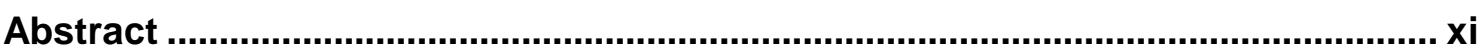

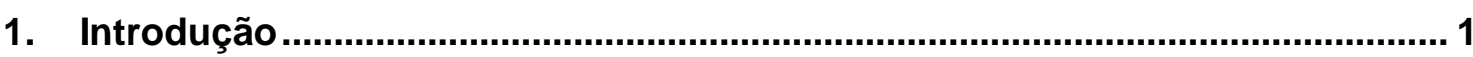

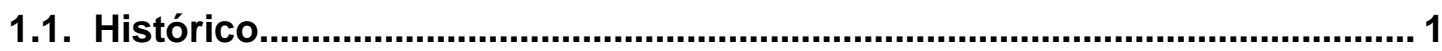

1.2. 0 estudo da relação entre o sono e a epilepsia ............................................ 2

1.2.1. O comportamento da atividade epileptiforme durante o sono ..............3

1.2.2. A influência dos distúrbios do sono sobre a epilepsia ...........................

1.2.3. A influência da epilepsia sobre a arquitetura do sono............................4

1.2.4. Epilepsia e o ciclo sono-vigília.................................................................

1.3. Qualidade de vida e funcionamento social ................................................. 7

1.3.1. Qualidade de vida ...............................................................................

1.3.2. Funcionamento social .........................................................................

1.4. Fatores que interferem na relação entre o sono e a epilepsia..................... 10

1.4.1. A influência dos fármacos antiepilépticos .............................................10

1.4.2. Transtornos do humor $e$ ansiedade ........................................................11

1.5. Resposta farmacológica .............................................................................. 13

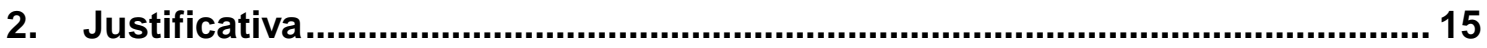

2.1. Os aspectos do sono em pacientes com epilepsia farmacorresistente.... 15

2.2. Os aspectos do sono e a qualidade de vida e o funcionamento social .... 16

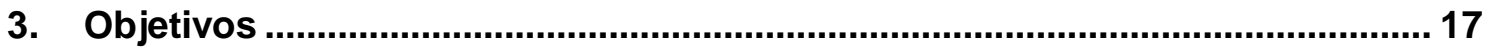

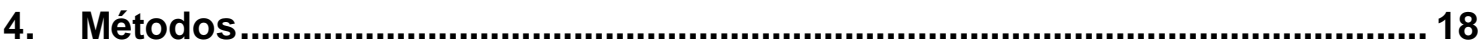

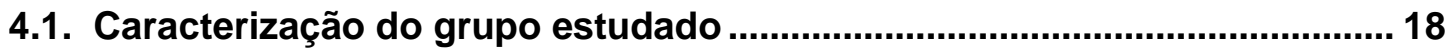

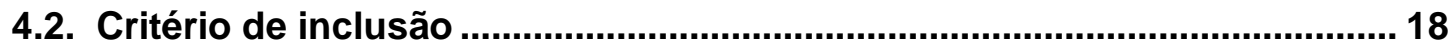

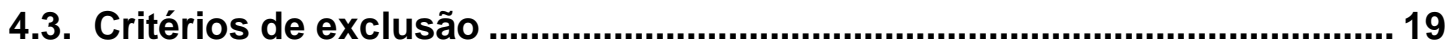

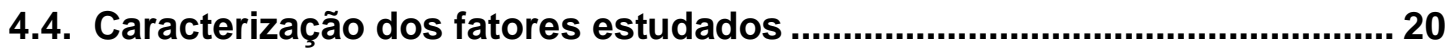

4.4.1. Caracterização da epilepsia ...................................................................

4.4.2. Cronotipo

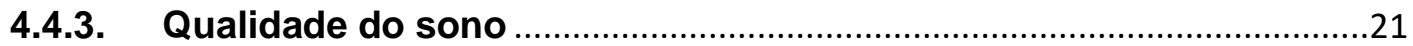

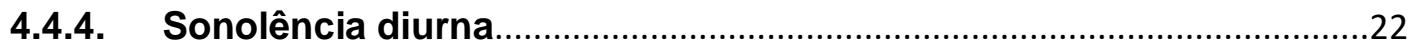




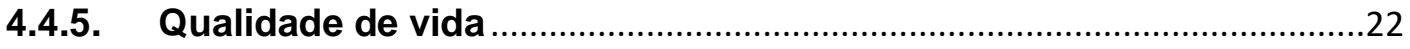

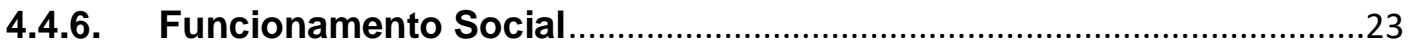

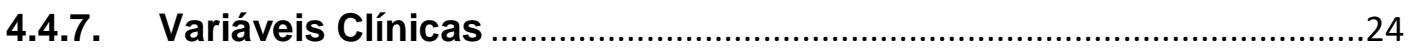

4.5. Análise Estatística ........................................................................................ 27

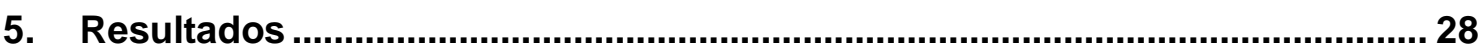

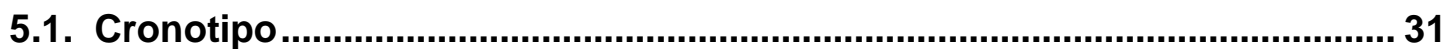

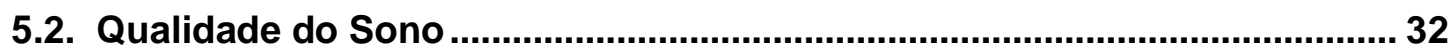

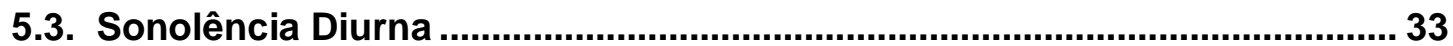

5.4. Relação entre os fatores relacionados à epilepsia e o Cronotipo, a

Qualidade de Sono e a Sonolência Excessiva Diurna ................................. 34

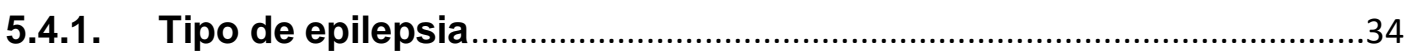

5.4.2. Número de Fármacos Antiepilépticos …................................................35

5.4.3. Frequência das Crises Epilépticas ............................................................

5.5. Relação entre o Cronotipo, a Qualidade de Sono e a Sonolência Excessiva Diurna com a Qualidade de Vida e a Adequação Social............................... 37

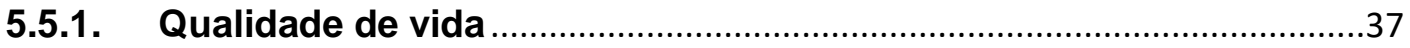

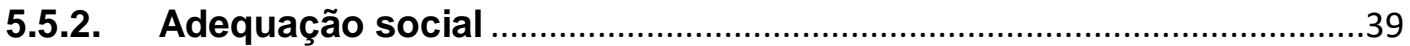

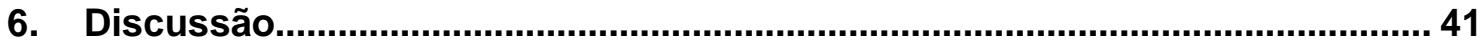

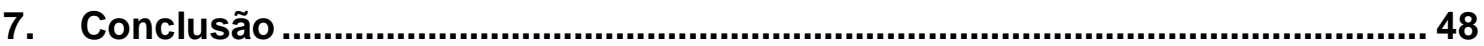

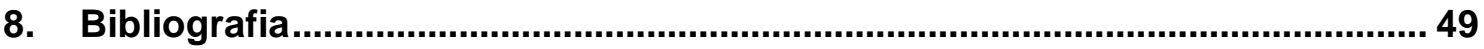

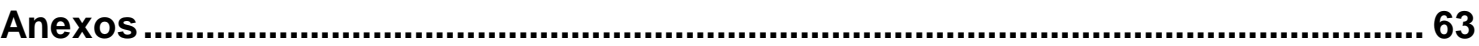




\section{Resumo}

Pentagna A. Os aspectos do sono nos pacientes com epilepsia farmacorresistente e seu impacto sobre a qualidade de vida e o funcionamento social [dissertação]. São Paulo: Faculdade de Medicina, Universidade de São Paulo; 2018.

Pacientes com epilepsia apresentam comorbidades que podem afetar a sua qualidade de vida e a sua adequação social. Por esta razão há um interesse crescente pelo estudo das condições associadas à epilepsia. Pacientes com epilepsia apresentam maior risco de desenvolver transtornos de sono. Entretanto, a relação entre o sono e a epilepsia permanece negligenciada. Os objetivos deste estudo foram: (i) avaliar o cronotipo, a presença de sonolência diurna e a qualidade do sono, através de medidas paramétricas, comparando pacientes farmacorresistentes e farmacossensíveis, e (ii) verificar a associação entre estes fatores e a qualidade de vida e o funcionamento social. Para tal, nós entrevistamos 114 pacientes (58 pacientes com epilepsia farmacossensível e 56 pacientes farmacorresistentes) e 62 indivíduos saudáveis sem epilepsia. Os grupos foram pareados por idade e sexo Todos os indivíduos entrevistados foram submetidos a questionários de autopreenchimento que verificam 0 cronotipo (Questionário de Matutinidade e Vespertinidade), a qualidade do sono (Índice de Qualidade de Sono de Pittsburgh) e a sonolência diurna (Escala de Sonolência de Epworth), bem como a qualidade de vida (Inventário de Qualidade de Vida em Epilepsia, versão com 31 perguntas) e o funcionamento social (Escala de Adequação Social). Também foram analisadas outras variáveis relacionadas à epilepsia como tipo de epilepsia (focal ou generalizada), quantidade de fármacos utilizados (monoterapia ou politerapia) e frequência de crises epilépticas. A caracterização das variáveis que podem interferir na análise das variáveis de desfecho foi realizada através da mensuração dos efeitos adversos de fármacos antiepilépticos (Perfil de Eventos Adversos de Liverpool), dos sintomas depressivos (Inventário de Depressão de Beck $2^{\mathrm{a}}$ versão) e dos sintomas de ansiedade (Inventário de Ansiedade Traço-Estado). Os grupos não diferiram quanto ao cronotipo e à sonolência diurna. Foi observada uma diferença significativa no resultado do PSQI do grupo farmacorresistente refletindo melhor qualidade de sono em relação aos outros dois grupos (diferença farmacossensível $x$ farmacorresistente 1,90, $p=0,003$; diferença farmacorresistente $x$ controle saudável $-1,53, p=0,041$ ). Em relação às variáveis clínicas, pacientes com epilepsia generalizada apresentaram maior vespertinidade do que os pacientes com epilepsia focal (diferença 11,99, $\mathrm{p}=0,0002$ ). Pacientes em monoterapia, avaliados pelo PSQI, apresentaram pior 
qualidade de sono (diferença $1,75, p=0,01$ ). A frequência de crises epilépticas não influenciou o resultado de cronotipo, qualidade de sono ou sonolência diurna. A qualidade de sono apresentou uma tendência à correlação com a qualidade de vida $(p=0,070)$. Houve uma associação entre o cronotipo e a adequação social $(p=0,027)$, porém apenas os farmacossensíveis se diferenciaram do grupo controle $(p=0,011)$, apresentando um pior funcionamento social relacionado com a vespertinidade. Os nossos resultados sugerem que a relação entre o sono e a epilepsia está mais intimamente ligada ao tipo de epilepsia, independente da resposta terapêutica ou da frequência de crises epilépticas. Além do mais, esta relação está ligada ao cronotipo, não à qualidade do sono ou à sonolência diurna. A qualidade de vida e a adequação social também estão relacionadas aos fatores relacionados ao sono.

Descritores: epilepsia; epilepsia resistente a medicamentos; sono; qualidade de vida; ajustamento social. 


\section{Abstract}

Pentagna A. Sleep aspects in patients with drug resistant epilepsy and their impact on quality of life and social adjustment [dissertation]. São Paulo: "Faculdade de Medicina, Universidade de São Paulo"; 2018.

Patients with epilepsy have comorbidities that may compromise their quality of life and social adjustment. For this reason, there is a growing interest in the study of conditions associated with epilepsy. Patients with epilepsy are at higher risk to develop sleep disorders. However, the relationship between sleep and epilepsy remains neglected. This study aimed to: (i) evaluate the chronotype, daytime sleepiness, and sleep quality by parametric measures, comparing patients with drug-responsive and drug-resistant epilepsy, and (ii) verify the association between these factors and quality of life and social functioning. For this purpose, we interviewed 114 patients (58 patients with drug-responsive epilepsy, and 56 patients with drug-resistant epilepsy) and 62 healthy controls without epilepsy. The groups were matched for age and gender. All individuals were interviewed with self-applied questionnaires that verified the chronotype (MorningnessEveningness Questionnaire), sleep quality (Pittsburgh Sleep Quality Index) and daytime sleepiness (Epworth Sleepiness Scale), as well as quality of life (Quality of Life Inventory in Epilepsy, 31 questions version) and social functioning (Social Adjustment Scale). We also evaluated other epilepsy-related variables, such as the epilepsy type (focal or generalized), number of antiepileptic medications (mono or polytherapy), and seizure frequency. The characterization of variables that can influence the analysis of the outcome was measured by the adverse effects of antiepileptic drugs (Liverpool Adverse Effects Profile), depressive symptoms (Beck Depression Inventory $2^{\underline{a}}$ version) and anxiety symptoms (State and Trait Anxiety Inventory). The groups did not differ in chronotype or daytime sleepiness. The drug-resistant group showed a better sleep quality compared to others (difference drug-responsive vs. drug-resistant 1.90, $\mathrm{p}=0.003$; difference drug-resistant vs. healthy controls $-1.53, \mathrm{p}=0.041$ ). Considering the clinical variables, patients with generalized epilepsy showed a more evening-oriented chronotype than patients with focal epilepsy (difference 11.99, $p=0.0002$ ). Patients under monotherapy, evaluated by PSQI, showed worse sleep quality (difference 1.75, $\mathrm{p}=0.01$ ). Seizure frequency did not influence the results for chronotype, sleep quality or daytime sleepiness presented a correlation with quality of life $(p=0,070)$. Sleep quality demonstrated a trend towards an association with quality of life $(p=0,070)$. There was an association between chronotype and social functioning $(p=0,027)$, however, only drug- responsive patients differed from controls without epilepsy $(p=0,011)$ with a worse social 
functioning that was eveningness-related. Our results suggest that the relationship between sleep and epilepsy is closely related to epilepsy type, regardless of therapeutic or seizure frequency. Additionally, it is related to the chronotype, but not to the quality of sleep or daytime sleepiness. Sleep quality is also associated with sleep-related factors.

Descriptors: epilepsy; drug resistant epilepsy; sleep; quality of life; social adjustment. 


\section{Introdução}

\subsection{Histórico}

A relação entre a epilepsia e o sono passou a ser objeto de estudo no final do século XIX com as observações feitas por Féré (1880), Gowers (1885) e Charcot (1892) (revisão em 1). Nestes estudos, foi observado um padrão de "eventos epilépticos" em horários determinados, destacando-se os períodos logo após o despertar e ao adormecer.

No século seguinte, houve um delineamento mais preciso sobre a associação entre a epilepsia e o sono. Em 1931, Party descreveu que os pacientes com crises de predomínio durante o sono tinham um horário de pico para as crises em duas horas após o adormecer ou uma hora antes do despertar; já os pacientes com padrão de crise na vigília apresentavam eventos em três picos de horário: logo após o despertar matutino, após o cochilo vespertino e durante a noite(revisão em 1).

Em 1962, Janz $(2,3)$ foi pioneiro ao criar uma classificação para os horários de ocorrência das crises epilépticas e sua correlação com a etiologia da epilepsia. As epilepsias foram classificadas em: epilepsias do despertar, noturnas e difusas, conforme o predomínio do horário de ocorrência das crises epilépticas associadas a outras características particulares que as acompanhavam. As epilepsias do despertar eram geralmente de etiologia indeterminada, iniciavam-se entre 10 e 25 anos e tenderiam a ocorrer de forma intermitente, sendo desencadeadas pela privação do sono ou consumo de 
álcool. As epilepsias noturnas, não relacionadas à faixa etária, eram caracterizadas por crises tônico-clônicas generalizadas (TCG) com origem predominantemente temporal, sendo que $60 \%$ delas apresentavam alguma lesão estrutural. As epilepsias difusas não teriam qualquer padrão de horário e as crises tônico-clônicas generalizadas eram geralmente lesionais e associadas a crises disperceptivas (previamente conhecidas como crises psicomotoras). Embora esta nomenclatura e esta classificação não tenham sido utilizada, descreve alguns tipos de epilepsia atualmente reconhecidos pela International League Against Epilepsy (ILAE) (4). Destaca-se o grupo das epilepsias do despertar que representam o protótipo do paciente com epilepsia mioclônica juvenil (EMJ) ao qual este autor posteriormente denominou de petit mal impulsiv (5).

\subsection{O estudo da relação entre o sono e a epilepsia}

A relação entre o sono e a epilepsia pode ser abordada de formas distintas:

a. o comportamento da atividade epileptiforme durante o sono (6-10);

b. a influência dos distúrbios do sono sobre a epilepsia (11-15);

c. a influência da epilepsia sobre a arquitetura do sono, num sentido inverso ao modelo anterior, com o objetivo de compreender como a epilepsia influenciaria o sono através de estudos neurofisiológicos (16-19) e;

d. a epilepsia e o cronotipo, que representa os estudos mais recentes, com o objetivo de identificar possíveis fenótipos circadianos dos pacientes com epilepsia (20-25). 


\subsubsection{O comportamento da atividade epileptiforme durante o sono}

A partir da década de 90 , estudos documentaram o comportamento da atividade epileptiforme na vigília e no sono nas diferentes fases do sono.

Adachi et al. (6) descreveram que na epilepsia do lobo temporal, a atividade epileptiforme documentada durante o sono tem maior valor localizatório. A melhor fase do sono para esta documentação permanece controversa. $\mathrm{O}$ estudo conduzido por Clemens et al. (7) sugere que há maior frequência da atividade epileptiforme durante o estágio N3. Sammaritano et al. (8) demonstraram que um delineamento mais preciso da zona epileptogênica poderia ser realizado durante o sono REM. Posteriormente, Bazil et al. $(9,10)$ observaram uma maior frequência de generalização de crises epilépticas focais quando estas ocorreram durante os estágios $\mathrm{N} 1$ e N2 nos pacientes com epilepsia do lobo temporal. Estes autores também relataram menor ocorrência de crises epilépticas durante o sono REM e a maior frequência de eventos durante o período noturno na epilepsia do lobo frontal.

\subsubsection{A influência dos distúrbios do sono sobre a epilepsia}

Posteriormente aos estudos neurofisiológicos da década de 90, a influência dos distúrbios do sono sobre a epilepsia passou a ser melhor estudada, predominantemente pela observação de que as crises epilépticas eram agravadas nos pacientes com epilepsia que apresentavam apneia obstrutiva do sono (AOS). Os dados sobre a prevalência da AOS entre os pacientes com epilepsia são discordantes, desde resultados semelhantes à 
população geral, ou seja, 10,2\% (11) até resultados elevados como 33\% (12). Apesar desta discordância, há evidências de que a adesão ao tratamento com pressão aérea positiva contínua (CPAP) para os pacientes que apresentam epilepsia e AOS pode levar à redução da frequência de crises pois reduzem a privação do sono, a exposição à hipóxia e ao estresse físico $(13,14)$.

Em relação à insônia, García-Morales et al. (15) compararam as Escalas de Sonolência de Epworth e a Escala de Insônia de Atenas em 237 pacientes com epilepsia focal farmacossensível e 264 pacientes com epilepsia focal farmacorresistente e observaram que $45 \%$ dos pacientes farmacorresistentes apresentavam algum distúrbio do sono comparados a $22 \%$ dos pacientes farmacossensíveis. Além disso, evidenciou-se a associação entre uma pior qualidade de vida, com maior ocorrência de insônia e sonolência excessiva diurna em farmacorresistentes.

\subsubsection{A influência da epilepsia sobre a arquitetura do sono}

A influência da epilepsia na arquitetura do sono foi melhor caracterizada no século XXI. Yu-Dan et al. (16) demonstraram que os pacientes com epilepsia passam mais tempo nos estágios $\mathrm{N} 1$ e $\mathrm{N} 2$ e menos tempo no estágio N3.

Krishnan et al. (17), através da análise polissonográfica de 25 pacientes com epilepsia mioclônica juvenil, descreveram que estes pacientes apresentam menor eficiência de sono, latência de sono aumentada, maior tempo em sono não REM N2 e maior número de microdespertares. Estas 
características sugerem que estes pacientes podem apresentar pior qualidade de sono e sintomas diurnos de sonolência excessiva, também descritas por outros autores $(18,19)$.

\subsubsection{Epilepsia e o ciclo sono-vigília}

Circadiano é uma palavra de origem latina que significa "por cerca de um dia" (circa+dies). O ritmo circadiano é o processo biológico de controle endógeno das oscilações fisiológicas e comportamentais dos organismos dentro do período de um dia (26). Essas variações são influenciadas por expressão gênica (clock genes), secreção hormonal e atividade neuronal, porém são "ajustadas" pelos fatores externos como a exposição à luminosidade, a atividade física e os hábitos cotidianos, como a alimentação (27). A ocorrência das crises epilépticas e o próprio estado de epilepsia poderiam influenciar nas variações do ritmo circadiano $(28,29)$. Poucos estudos abordaram o ciclo sono-vigília dos pacientes com epilepsia e como seu quotidiano pode ser influenciado por esta condição (20-22). Estes estudos buscaram caracterizar cronotipos, ou seja, fenótipos circadianos de preferência para os horários de sono e atividade que possam se correlacionar à epilepsia. Os cronotipos são divididos em vespertinos, que tendem a deitar tarde e acordar tarde, matutinos, que deitam cedo e despertam cedo e intermediários, que correspondem ao padrão principal e geralmente representam a preferência de horários(30).

Desde a descrição de Janz (revisão em 1) sobre os pacientes com epilepsia mioclônica juvenil, existe o conceito de que estes pacientes apresentem atraso da fase de sono (deitam mais tarde e levantam mais tarde). 
As crises epilépticas, principalmente generalizadas, provocam picos de prolactina, noradrenalina, vasopressina e ocitocina, hormônios que têm um padrão circadiano de secreção. Apesar de controverso, alguns autores sugerem que as crises epilépticas "reiniciariam" um ritmo circadiano ou mesmo alterariam a fase. Outra hipótese seria de que a epilepsia, a longo prazo, comprometeria as vias relacionadas ao ritmo circadiano(31).

Pung e Schmitz (23) utilizaram o questionário de matunidade e vespertinidade de Horne e Östberg (Morningness-Eveningness Questionnaire MEQ) para 20 pacientes com diagnóstico de epilepsia mioclônica juvenil e confirmaram a tendência de deitar-se e levantar-se mais tarde destes indivíduos, com melhor sensação de bem-estar ao longo do dia se comparados com um grupo de pacientes com epilepsia do lobo temporal.

Já Hofstra et al. (24) aplicaram o MEQ e o Munich Chronotype Questionnaire (MCTQ) a um grupo de 200 pacientes com epilepsia e observaram a tendência de um padrão de avanço da fase de sono. Estes autores abordaram três grupos específicos - epilepsia mioclônica juvenil, epilepsias do lobo temporal e do lobo frontal. Em oposição ao observado por Pung e Schmitz (23), os pacientes com epilepsia mioclônica juvenil não diferiram dos demais.

Os hábitos de sono de 121 crianças com epilepsia foram analisados por Batista e Nunes (25) através do Sleep Habits Inventory for Preschool Children e do Sleep Behavior Questionnaire estratificados conforme a faixa etária. Estes autores observaram que as crianças com crises epilépticas noturnas, crises generalizadas, epilepsia farmacorresistente, atraso no 
desenvolvimento e com síndromes epilépticas de pior prognóstico apresentaram hábitos de sono piores.

\subsection{Qualidade de vida e funcionamento social}

A relação entre o sono e a qualidade de vida e o funcionamento social é pouco estudado e controverso.

\subsubsection{Qualidade de vida}

O estudo de de Weerd et al. (32), com 486 pacientes com epilepsia focal, avaliados através de diversos questionários (WHO Epilepsy Questionnaire, Sleep Diagnosis List - Sleep Diagnosis Questionnaire, Medical Outcomes Study - Sleep Scale, Groningen Sleep Questionnaire, Epworth Sleepniness Scale e SF-36 Health Survey), observou que os pacientes com epilepsia apresentam um risco duas vezes maior que o grupo controle de apresentar algum distúrbio do sono e sugerem que isto estaria implicado na pior qualidade de vida que estes indivíduos apresentam(33). Alanis-Guevara et al. (32) analisaram a qualidade de vida em 401 pacientes com epilepsia através do QOLIE-31 e evidenciaram que os fatores de maior influência para pior qualidade de vida foram a presença de distúrbios do sono, pior situação socioeconômica, o sexo feminino e uma maior ocorrência de crises epilépticas. Esses autores não evidenciaram que a depressão e a cronicidade da doença tivessem influência sobre a qualidade de vida. Piperidou et al. (34) aplicaram o QOLIE-31, a Escala de Sonolência de Epworth, a Escala de Insônia de Atenas e a Escala de Apneia do Sono em 124 
pacientes e observaram que a insônia foi um fator determinante na pior qualidade de vida. Kwan et al. (35) analisaram o HADS, o Hamilton Anxiety Rating Scale, o Medical Outcomes Study - Sleep Scale, a Escala de Sonolência de Epworth e o QOLIE-31 em 247 pacientes com epilepsia e determinaram que os sintomas de depressão e ansiedade, além dos distúrbios do sono, foram os fatores de maior influência na qualidade de vida.

Hermann et al. (36) compararam testes de qualidade de vida (QOLIE89) e sintomas psiquiátricos (SCL-90-R - Symptom Checklist-90-Revised), em 54 pacientes com epilepsia do lobo temporal e 38 indivíduos saudáveis, relatando pior qualidade de vida e maiores escores de sintomas psiquiátricos nos pacientes com epilepsia, relacionadas com a cronicidade da doença. Johnson et al. (37) avaliaram as características clínicas (Liverpool Seizure Severity Scale), sintomas interictais de depressão e ansiedade (SCL-90-R e Inventário de Depressão de Beck), bem como a qualidade de vida (QOLIE-89), de 87 pacientes com epilepsia do lobo temporal e demonstraram que os sintomas depressivos e de ansiedade tiveram maior associação com piores escores de qualidade de vida do que a frequência das crises epilépticas, a gravidade delas e a duração da doença.

No entanto, Jacoby et al. (38), após a análise de questionários aplicados a 947 pacientes com epilepsia (State-Trait Anxiety Scale, Pittsburgh Sleep Quality Index, Escala de Sonolência de Epworth, Perfil de Efeitos Adversos de Liverpool, Escala de Impacto da Epilepsia, Seizure Worry Scale, Social Support Scale, 3-Item Epilepsy-Specific Scale) não obtiveram essa correlação de influência direta entre a má qualidade do sono e uma pior qualidade de vida 
na epilepsia apesar de afirmarem que os efeitos adversos das medicações tiveram influência sobre o sono destes pacientes.

\subsubsection{Funcionamento social}

Há poucos estudos abordando o funcionamento social nos pacientes com epilepsia. Moschetta e Valente (39) aplicaram o SAS (Self-Report Social Adjustment Scale) em um grupo de 42 pacientes com epilepsia mioclônica juvenil e observaram que um pior ajustamento social nas relações familiares e de situação laboral esteve relacionado a uma personalidade impulsiva e maior frequência de crises, mas não a um pior desempenho cognitivo. Gois et. al (40), através do uso do mesmo questionário SAS, demonstraram que em 35 pacientes com epilepsia do lobo temporal por esclerose hipocampal houve uma pior adequação social relacionada aos seus déficits cognitivos. Isto ressalta a importância da abordagem individualizada das síndromes epilépticas.

Gutter, Brouwer e de Weerd (41) fizeram uma análise similar em uma população pediátrica através do Medical Outcomes Study - Sleep Scale, o Groningen Sleep Scale, o Hague Scale (para aferir a gravidade da epilepsia) e o Kidscreen-27 (para aferir a qualidade de vida). Entre as 130 crianças com epilepsia focal avaliadas houve uma prevalência de $37 \%$ de anormalidade nos escores de distúrbio do sono comparados aos 3\% observados nas 166 crianças saudáveis do grupo controle. As crianças com epilepsia e pior qualidade de sono apresentaram pior desempenho no ambiente escolar e no bem-estar físico e psicológico quando comparadas com as crianças com epilepsia e sem distúrbios de sono. 
Ainda não foi avaliada a relação entre os diferentes aspectos do sono (qualidade do sono, sonolência diurna e cronotipo) com o funcionamento social nos pacientes com epilepsia.

\subsection{Fatores que interferem na relação entre o sono e a epilepsia}

A relação entre o sono e a epilepsia pode ser influenciada por duas principais condições: os efeitos adversos dos fármacos antiepilépticos (FAE) e a presença de transtornos psiquiátricos. A relevância destes fatores deve ser compreendida, visto que estes podem ser fatores confusionais no estudo da associação entre o sono e a epilepsia.

\subsubsection{A influência dos fármacos antiepilépticos}

Os efeitos dos FAE sobre o sono e os sintomas diurnos relacionados ao sono são bem conhecidos. Placidi et al. (42) evidenciaram que a carbamazepina na sua apresentação de liberação controlada causa fragmentação apenas na introdução do medicamento, e a lamotrigina e a gabapentina promovem certa estabilização do sono. Legros e Bazil (43) realizaram 72 polissonografias em 39 pacientes e observaram um aumento da quantidade de sono de ondas lentas (estágio N3) nos pacientes em uso da

gabapentina; aumento da porcentagem de sono N1 com o ácido valproico e a fenitoína, sendo o último também causador de redução da fase N3 do sono NREM. Por outro lado, a carbamazepina e a lamotrigina não demonstraram 
efeitos relevantes. Portanto, diferentes FAE apresentam impactos distintos sobre a arquitetura do sono.

Além da análise polissonográfica, Salinsky et al. (44), utilizando o teste de manutenção da vigília (TMV), nos pacientes em uso de carbamazepina, ácido valproico, fenobarbital e fenitoína, observaram que pacientes em uso de FAE apresentam maior dificuldade para se manterem despertos comparados aos controles (indivíduos sem transtornos neurológicos ou psiquiátricos, pacientes com esclerose múltipla e pacientes com epilepsia sem tratamento médico).

O conhecimento sobre o impacto dos fármacos no dia-a-dia é importante, visto ser o tratamento medicamentoso parte da premissa destes pacientes. Hoje, a relação entre os efeitos adversos e o padrão de sono pode ser avaliada de forma paramétrica através de escalas objetivas.

\subsubsection{Transtornos do humor e ansiedade}

Os transtornos do humor ocorrem em $11 \%$ a $62 \%$ dos pacientes com epilepsia, sendo predominante o transtorno depressivo (20\% a $55 \%)(45)$. Nos pacientes com epilepsia famacorresistente, a presença e a gravidade de transtornos de humor são tão relevantes quanto a frequência de crises na determinação da qualidade de vida $(35,46,47)$. A detecção dos transtornos do humor é essencial, dada a sua prevalência elevada nesta população, e o seu impacto sobre a qualidade do sono. 
Elsharkawy el al. (48) avaliaram, através de questionários, 359 pacientes com epilepsia focal farmacorresistente. Cento e vinte e cinco pacientes responderam o QOLIE-31 (Qualidade de Vida em Epilepsia), o HADS (Hospital Anxiety and Depression Scale) e o questionário PESOS (Performance Sociodemographic Aspects), que avalia parâmetros sociais e ocupacionais, bem como os efeitos do tratamento da epilepsia quanto à eficácia e aos efeitos adversos descritos de forma subjetiva pelos próprios pacientes. Estes autores observaram que a ansiedade e a depressão são importantes variáveis para a determinação da qualidade de vida, mas não são as únicas; a situação de desocupação laboral, os efeitos adversos das medicações e a frequência de crises epilépticas também foram determinantes na qualidade de vida.

Pompili et al. (49) compararam os resultados do Inventário de Depressão de Beck II, a Escala de Desesperança de Beck e o QOLIE-89 entre pacientes com crises epilépticas generalizadas e pacientes com crises focais. Demonstrou-se que todos os 69 participantes apresentavam depressão moderada a grave e que a ocorrência de crises epilépticas generalizadas (35,4\% dos pacientes) foi determinante para uma maior dificuldade no exercício de atividades sociais como consequência dos problemas emocionais. Além disso, os autores também descreveram que $25 \%$ dos pacientes apresentaram uma pontuação $\geq 9$ na Escala de Desesperança de Beck, determinando um maior risco para suicídio. Em outra análise de 33 pacientes com epilepsia mioclônica juvenil, sendo 21 mulheres, em acompanhamento há mais de 20 anos num centro terciário de atendimento de epilepsia observou-se que a não ocorrência de crises é fator de melhor qualidade de vida enquanto que uma maior gravidade 
da doença, os efeitos adversos das medicações, a presença de depressão e o comprometimento do sono são determinantes de uma pior qualidade(50). Neste estudo foram utilizados o QOLIE-31 e o Inventário de Depressão de Beck II, além de perguntas diretas sobre adequação social. Particularmente para a adequação social, consideraram-se como deficiências o desemprego, a mudança de trabalho devido à epilepsia, dificuldades na formação profissional, evasão escolar, penalização por má conduta na escola, repetência, condenação por crime, gravidez não planejada, abortamento provocado pelo fato de apresentar epilepsia, ausência de relacionamentos por mais de três meses, celibatarismo e divórcio. Ao menos uma dessas carências sociais foi encontrada em $87,9 \%$ dos pacientes, destacando-se a gravidez não planejada (36,2\%), o celibatarismo $(27,3 \%)$ e o desemprego $(26,3 \%)$.

\subsection{Resposta farmacológica}

Segundo a International League Agaisnt Epilepsy (ILAE), a epilepsia farmacorresistente é caracterizada pela persistência das crises epilépticas apesar do uso de dois esquemas de tratamento diferentes, seja monoterapia ou politerapia (dois ou mais medicamentos), que tenham sido bem tolerados e escolhidos de forma adequadamente direcionada à etiologia da epilepsia a ser tratada (51). Estes pacientes correspondem a até $40 \%$ dos portadores de epilepsia (52) e são responsáveis por $80 \%$ dos custos de tratamento da doença nos Estados Unidos (53). Além disso, causa impacto social significativo e aumenta a mortalidade em 5 a 10 vezes mais que na população sem epilepsia devido a morte súbita, acidentes ou suicídio (54). 
Classicamente, o abandono do tratamento ou uso irregular dos FAE, geralmente provocados por intolerância aos efeitos adversos, interações farmacológicas, privação de sono, cansaço e estresse psíquico são os principais fatores desencadeantes das crises epilépticas (55-57).

Pela sua própria definição, os pacientes farmacorresistentes estão mais susceptíveis à condição de politerapia (mais de um fármaco) e à persistência das crises epilépticas (51). Portanto, esta é uma população com risco aumentado de eventos adversos de fármacos, sintomas depressivos e ansiosos, todas condições que poderiam interferir significativamente no sono destes indivíduos (58) e, já sabidamente, na qualidade de vida (46-48). No entanto, é desconhecido como os aspectos do sono se apresentam nestes indivíduos e como eles se correlacionam à qualidade de vida e ao funcionamento social sem a influência dos eventos adversos, os sintomas depressivos e ansiosos. 


\section{Justificativa}

Dentro do contexto atual de "tratar além das crises epilépticas", o estudo das comorbidades é fundamental. Apesar disso, o estudo do sono nos pacientes com epilepsia permanece negligenciado.

As características do cronotipo, bem como a qualidade de sono e a sonolência diurna, podem ser abordados através de questionários padronizados para este fim.

\subsection{Os aspectos do sono em pacientes com epilepsia}

\section{farmacorresistente}

Há poucas evidências das alterações do cronotipo, qualidade de sono e sonolência diurna nos pacientes com epilepsia farmacorresistente. Os fatores determinantes da pior qualidade de sono e das alterações do ritmo circadiano permanecem controversos. A determinação de fatores preditivos destas alterações é relevante a fim de que possamos determinar subgrupos de risco para as alterações do sono.

É razoável supor que pacientes com epilepsia farmacorresistente apresentem pior qualidade do sono quando comparados a indivíduos saudáveis e indivíduos com epilepsia farmacossensível. O mesmo não pode ser afirmado para o cronotipo, pois pacientes com epilepsia generalizada farmacossensível parecem apresentar maior vespertinidade. Portanto, há poucos estudos sobre 
estas características (cronotipo, qualidade de sono e sonolência diurna) nos pacientes farmacorresistentes comparados aos farmacossensíveis, sendo este o objetivo primário do presente estudo.

\subsection{Os aspectos do sono e a qualidade de vida e o funcionamento} social

A maior dificuldade em avaliar o impacto do sono sobre a qualidade de vida e o funcionamento social está relacionada à interação entre diferentes fatores, dentre elas a etiologia da síndrome epiléptica, a frequência das crises, as comorbidades psiquiátricas e os efeitos adversos dos fármacos antiepilépticos.

O estudo de Utenberger et al. (2015) não demonstrou correlação entre o cronotipo dos pacientes com epilepsia e a qualidade de vida (21). Poucos estudos foram realizados com o objetivo de demonstrar o quanto a pior qualidade do sono influencia na qualidade de vida deste grupo de pacientes $(15,59,60)$. Além disso, nenhum trabalho demonstrou a relação entre a qualidade de sono e a adequação social desses indivíduos, avaliada através de uma escala objetiva e paramétrica como realizado pelo nosso grupo anteriormente para o estudo da cognição e transtornos psiquiátricos $(39,40)$.

O entendimento do impacto do cronotipo, da qualidade de sono e da sonolência diurna sobre a qualidade de vida e o funcionamento social é o segundo objetivo do estudo. 


\section{Objetivos}

Este estudo transversal e controlado teve como objetivos:

3.1. Avaliar o cronotipo, a qualidade de sono e a sonolência diurna nos pacientes com epilepsia farmacorresistente, comparados a pacientes com epilepsia farmacossensível e a controles sem epilepsia;

3.2. Determinar a relevância das variáveis relacionadas à epilepsia: frequência das crises epilépticas, a etiologia da epilepsia (focal ou generalizada) e o uso de fármacos antiepilépticos nas possíveis alterações do ritmo circadiano, da qualidade de sono e da sonolência diurna;

3.3. Analisar o impacto das alterações do cronotipo, qualidade de sono e sonolência diurna no funcionamento social e na qualidade de vida. 


\section{Métodos}

\subsection{Caracterização do grupo estudado}

\section{a. Pacientes}

Os pacientes foram caracterizados quanto aos fatores demográficos: idade, sexo, situação laboral, nível educacional e nível sócioeconômico, sendo provenientes do Ambulatório de Epilepsia do Instituto de Psiquiatria do Hospital das Clínicas da Faculdade de Medicina da Universidade de São Paulo.

\section{b. Indivíduos saudáveis sem epilepsia}

Foram avaliados indivíduos sem epilepsia, pareados em relação à idade e ao sexo. Estes indivíduos foram recrutados entre os acompanhantes dos pacientes e voluntários. Os mesmos critérios de inclusão (excetuando-se o item 1) e exclusão foram aplicados.

\subsection{Critério de inclusão}

Os pacientes incluídos neste estudo apresentaram:

a. Diagnóstico de epilepsia, segundo a definição operacional da $\operatorname{ILAE}(61)$;

b. Idade maior que ou igual à 18 anos;

c. Terem assinado e compreendido o termo de consentimento livre e esclarecido. 


\subsection{Critérios de exclusão}

Foram excluídos pacientes que preenchessem os seguintes critérios:

a. Pacientes que apresentassem quadros clínicos que impedissem a compreensão ou o autopreenchimento do questionário:

$\checkmark$ Síndrome demencial;

$\checkmark$ Deficiência intelectual;

$\checkmark$ Transtornos neurológicos ou mentais graves que impedissem 0 entendimento ou o ato de responder os questionários, como: estado psicótico, maniforme ou afasia.

b. Uso de substâncias que pudessem interferir no ritmo circadiano além dos fármacos utilizados entre pacientes com epilepsia:

$\checkmark$ História atual de abuso de álcool e drogas ilícitas;

$\checkmark$ História atual de uso de medicamentos com propriedades psicoestimulantes como modafinila e metilfenidato.

Além disso, também foram excluídos:

$\checkmark$ Trabalhadores de turno

$\checkmark$ Pacientes que realizassem viagens frequentes através de fusos horários diferentes (jet lag) 


\subsection{Caracterização dos fatores estudados}

\subsubsection{Caracterização da epilepsia}

Os pacientes foram classificados segundo a sua resposta ao tratamento em:

a. Pacientes com epilepsia farmacorresistente: pacientes adultos com epilepsia farmacorresistente em seguimento ambulatorial. O diagnóstico de farmacorresistência foi realizado através de dados clínicos de acordo com a proposta vigente da ILAE, ou seja, o não controle das crises epilépticas mesmo após o uso correto de dois esquemas terapêuticos tolerados e adequadamente escolhidos, seja em mono ou politerapia (51);

b. Pacientes com epilepsia farmacossensível: pacientes adultos com epilepsia controlada há pelo menos um ano em seguimento ambulatorial com perfis semelhantes ao do grupo de estudo, sendo pareados em relação à idade, ao sexo e à sua ocupação. Os mesmos critérios de inclusão (excetuandose o item 1) e exclusão serão aplicados.

Os pacientes estudados também foram avaliados quanto:

a. ao tipo de epilepsia: focal ou generalizada;

b. à quantidade de fármacos antiepilépticos em uso: monoterapia (um fármaco antiepiléptico em uso) ou politerapia (dois ou mais fármacos antiepilépticos em uso)

c. à frequência das crises epilépticas. 


\subsubsection{Cronotipo}

a. Questionário de matutinidade e vespertinidade (MorningnessEveningness Questionaire - MEQ)

O MEQ foi desenvolvido por Horne e Ostberg em 1976 (30), sendo traduzido para o português no Brasil (62). Trata-se de um questionário de autopreenchimento que determina qual o padrão de horário com melhor funcionamento de um indivíduo para suas atividades de vida diária e prática. A aplicação do questionário resulta em pontuação que divide os pacientes em grupos denominados como vespertino (16-41 pontos), intermediário (42-58 pontos) e matutino (59-86 pontos).

\subsubsection{Qualidade do sono}

a. Índice de Qualidade de Sono de Pittsburgh (Pittsburgh Sleep Quality Index - PSQI)

O PSQI foi desenvolvido por Buysse em 1989 (63), sendo traduzido e validado para o português no Brasil (64). É o inventário mais utilizado atualmente para a análise da qualidade de sono de um indivíduo baseado na sua própria percepção. O PSQI é um questionário que tem como fundamento uma análise quantitativa e qualitativa baseada no último mês e desta forma não ignora os problemas de sono tanto recentes e quanto antigos que influenciariam na qualidade de sono do indivíduo. É constituída por 19 questões respondidas pelo paciente e cinco direcionadas ao seu companheiro de quarto, sendo estas apenas para informação clínica. A pontuação varia de zero a 21 , sendo que 
escores maiores equivalem a pior a qualidade do sono. Índices acima de cinco pontos demonstram problemas relacionados à qualidade do sono.

\subsubsection{Sonolência diurna}

a. Escala de Sonolência de Epworth (Epworth Sleepiness Scale ESS)

O ESS foi desenvolvido por Johns em 1991 (65), sendo traduzido para o português no Brasil (66). Este questionário tem o objetivo de quantificar a intensidade de sonolência diurna referida. Oito situações cotidianas são apresentadas ao paciente que deve sugerir qual a possibilidade que ele tem de cochilar em cada uma delas. A graduação é feita por pontos sendo: 0 , nenhuma chance; 1 , chance pequena; 2 , chance moderada e 3 , grande chance de cochilar. A sonolência excessiva diurna é determinada por um escore superior ou igual a 10 .

\subsubsection{Qualidade de vida}

a. Inventário de qualidade de vida em epilepsia (Quality of Life Inventory in Epilepsy - QOLIE-31):

O QOLIE-31 foi criado por Cramer et al. em 1998 (67), sendo traduzido e validado para o português no Brasil (68). É o instrumento de avaliação da qualidade de vida nas pessoas com epilepsia mais utilizada na literatura mundial. Derivado do QOLIE-89, compreende as perguntas consideradas mais pertinentes pelos pacientes, é de fácil aplicação por ser de autopreenchimento e tem valor semelhante ao questionário mais extenso. São 
analisadas a preocupação do paciente em relação às crises epilépticas, a sensação de bem-estar, o cansaço e a volição, o funcionamento cognitivo, os efeitos medicamentosos e o funcionamento social. A nota varia numa escala de zero a cem pontos, sendo que escores mais elevados estão associados à melhor qualidade de vida. A escala de qualidade de vida para pacientes com epilepsia não pode ser aplicada à controles devido à especificidade das suas questões. Portanto, a escala de qualidade de vida pode ser comparada somente entre pacientes com epilepsia.

\subsubsection{Funcionamento Social}

\section{a. Escala de adequação social (EAS)}

A EAS foi desenvolvida por Weissman e Bothwell em 1976 (69), sendo validada e traduzida para a população brasileira (70). A escala avalia o desempenho instrumental e afetivo em áreas diversas: aspectos das relações interpessoais, atrito com os outros, sentimentos íntimos e insatisfação nos papéis. É composta por 54 questões de autopreenchimento, divididas em sete áreas específicas: trabalho, vida social e lazer, relação com a família, relação marital, relação com os filhos, vida doméstica e situação financeira. Os escores na EAS são obtidos para cada uma das sete áreas específicas, além de um escore global. Foi incluída a fim de melhor caracterizar a influência do sono sobre os pacientes, não apenas sobre a sua percepção de vida, reportado pelo questionário de qualidade de vida, como sobre o que ocorre de fato em seu cotidiano. Além disso, pode ser aplicado tanto para pacientes com epilepsia quanto para os voluntários sem epilepsia. 


\subsubsection{Variáveis Clínicas}

Devido ao impacto dos transtornos de humor, dos sintomas ansiosos e dos FAEs nas variáveis de desfecho, foram aplicados os inventários de Depressão de Beck II (BDI-II) e o de Ansiedade Traço-Estado (IDATE), além do Perfil de Eventos Adversos de Liverpool (LAEP).

a. Perfil de Eventos Adversos de Liverpool (Liverpool Adverse Events Profile - LAEP):

Foi desenvolvido por Baker et al. (71) em 1995, sendo traduzido e validado para seu uso no Brasil (72). O LAEP é composto pelos 19 sintomas adversos mais comuns entre usuários de fármacos antiepilépticos. O paciente deve pontuar de 1 (nunca) a 4 (sempre) em relação à frequência dos eventos adversos que ele sente. Seu resultado varia entre 19 e 76 , sendo que escores mais elevados representam maior impacto dos efeitos adversos.

b. Inventário de Depressão de Beck II (Beck Depression Inventory II - BDI II):

Esta escala de auto avaliação foi criada por Beck et al., em 1961 e atualizada em 1996 (73), sendo validada para o português no Brasil (74). É composto por 21 itens, cuja intensidade varia de zero a quatro. Estes itens são referentes aos seguintes sintomas depressivos: tristeza, pessimismo, sensação de fracasso, falta de satisfação, sensação de culpa, sensação de punição, autodepreciação, ideias suicidas, crises de choro, irritabilidade, retração social, indecisão, distorção da imagem corporal, inibição para o trabalho, distúrbio de sono, fadiga, perda de apetite, perda de peso, preocupação somática e diminuição da libido. O escore é obtido através da soma da pontuação obtida em 
cada um dos 21 itens, sendo que quanto maior o escore obtido, maior a expressão de sintomas depressivos.

\section{c. Inventário de Ansiedade Traço-Estado (IDATE)}

Foi desenvolvido por Spielberger et al. em 1970 (75), com validação para o português no Brasil (76). O IDATE é um instrumento de auto-avaliação de sintomas ansiosos, através da concepção dualística de ansiedade: ansiedadetraço (IDATE-T) e ansiedade-estado (IDATE-E) proposta inicialmente por Catell e Scheier, em 1961. O IDATE é composto por duas escalas: a) IDATE-T mede o traço de ansiedade, ou seja, uma condição mais estável de propensão à ansiedade, referente às características do indivíduo; b) IDATE-E mede o estado de ansiedade: estado emocional transitório ou condição do organismo humano caracterizada por sentimentos desagradáveis de tensão e apreensão, percebidos conscientemente e por aumento na atividade do sistema nervoso autônomo. Cada uma dessas escalas possui 20 itens que devem ser caracterizados através de parâmetros de intensidade que variam de um a quatro e somados para obtenção do escore final. Escores maiores indicam maior expressão de sintomas ansiosos.

As variáveis avaliadas e os respectivos métodos escolhidos estão demonstradas no Quadro 1. 
Quadro 1 - Resumo das variáveis a serem avaliadas e os respectivos métodos escolhidos

\begin{tabular}{|c|c|}
\hline Variável & Método \\
\hline \multicolumn{2}{|c|}{ Caracterização do padrão de sono } \\
\hline Padrão circadiano & Questionário de matutinidade e vespertinidade (MEQ) \\
\hline Qualidade do sono & Índice de Qualidade de Sono de Pittsburgh (PSQI) \\
\hline Sonolência diurna & Escala de sonolência de Epworth (ESS) \\
\hline \multicolumn{2}{|c|}{ Análise dos fatores relacionados à epilepsia } \\
\hline $\begin{array}{l}\text { Resposta ao } \\
\text { tratamento }\end{array}$ & Definida conforme a classificação da ILAE (51) \\
\hline Tipo de epilepsia & $\begin{array}{l}\text { Determinada através de história clínica, } \\
\text { eletroencefalograma e neuroimagem como } \\
\text { generalizada ou focal }\end{array}$ \\
\hline $\begin{array}{l}\text { Quantidade de } \\
\text { fármacos }\end{array}$ & $\begin{array}{l}\text { Monoterapia (uso de até } 1 \text { fármaco antiepiléptico) ou } \\
\text { politerapia (uso de } 2 \text { ou mais fármacos antiepilépticos) }\end{array}$ \\
\hline $\begin{array}{l}\text { Frequência de } \\
\text { crises epilépticas }\end{array}$ & Conforme relato do paciente farmacorresistente \\
\hline \multicolumn{2}{|c|}{ Análise dos fatores relacionados à vida } \\
\hline Qualidade de vida & $\begin{array}{l}\text { Inventário de qualidade de vida em epilepsia (QOLIE- } \\
\text { 31) }\end{array}$ \\
\hline Adequação social & Escala de adequação social (EAS) \\
\hline \multicolumn{2}{|c|}{ Variáveis de Confusão } \\
\hline $\begin{array}{l}\text { Efeitos colaterais } \\
\text { dos FAE }\end{array}$ & Perfil de Eventos Adversos de Liverpool (LAEP) \\
\hline $\begin{array}{l}\text { Sintomas } \\
\text { depressivos }\end{array}$ & Inventário de Depressão de Beck II (BDI-II) \\
\hline Sintomas ansiosos & Inventário de Ansiedade Traço-Estado (IDATE) \\
\hline
\end{tabular}




\subsection{Análise Estatística}

A análise estatística foi realizada com o uso da versão 3.5.1 do programa $\mathrm{R}$ de análise estatística (R Foundation@, Viena, Áustria).

Verificamos a relação entre as variáveis com os desfechos de interesse comparando os grupos e controlando pelas variáveis de confusão (Perfil de Eventos Adversos de Liverpool, segunda versão do Inventário de Depressão de Beck e Inventário de Ansiedade Traço-Estado) com modelos de regressão linear. A relação com a frequência das crises foi feita com correlação de Kendall.

Também foram realizados os contrastes dois a dois entre os grupos dentro das regressões lineares realizados através do método de Tukey para correção de erro tipo I associado a este processo.

Os dados obtidos foram considerados como significativos com um valor-p a partir de 0,05. 


\section{Resultados}

Dentre os 185 indivíduos convidados para o estudo, dois deles se recusaram a participar e sete não retornaram as solicitações. Ao todo, foram entrevistados 176 indivíduos (65,34\% sexo feminino; idade média 37,49 anos; DP $\pm 12,10$ ) entre julho de 2015 e junho de 2018. A distribuição dos indivíduos entrevistados por sexo e idade está demonstrada na Tabela 1. Os pacientes e os controles foram pareados para sexo $(p=0,704)$, idade $(p=0,747)$ e situação marital $(p=0,625)$.

As características dos pacientes farmacorresistentes e farmacossensíveis estão demonstradas nos Anexos 1 e 2.

Tabela 1 - Variáveis demográficas

\begin{tabular}{cccccc}
\hline & $n$ & \multicolumn{2}{c}{ Idade } & \multicolumn{2}{c}{ Sexo } \\
\hline & & Média & $\begin{array}{c}\text { Desvio } \\
\text { padrão }\end{array}$ & Feminino & Masculino \\
\hline $\begin{array}{c}\text { Controle sem } \\
\text { epilepsia }\end{array}$ & 62 & 35,53 & 11,21 & $\begin{array}{c}43 \\
(69,35 \%)\end{array}$ & $\begin{array}{c}19 \\
(30,65 \%)\end{array}$ \\
\hline Epilepsia & 114 & 38,56 & 12,48 & $\begin{array}{c}72 \\
(63,16 \%)\end{array}$ & $\begin{array}{c}42 \\
(36,84 \%)\end{array}$ \\
\hline Farmacossensível & 58 & 37,95 & 12,65 & $\begin{array}{c}37 \\
(63,79 \%)\end{array}$ & $\begin{array}{c}21 \\
(36,21 \%)\end{array}$ \\
\hline Farmacorresistente & 56 & 39,20 & 12,27 & $\begin{array}{c}35 \\
(62,50 \%)\end{array}$ & $\begin{array}{c}21 \\
(37,50 \%)\end{array}$ \\
\hline Total & 176 & 37,49 & 12,10 & $\begin{array}{c}115 \\
(65,34 \%)\end{array}$ & $\begin{array}{c}61 \\
(34,66 \%)\end{array}$ \\
\hline
\end{tabular}


Em relação à epilepsia, 90 pacientes $(78,95 \%)$ apresentavam epilepsia focal e 24 pacientes (21,05\%\%), epilepsia generalizada. A Tabela 2 demonstra a diferença entre os grupos de tipo de epilepsia, com predomínio das epilepsias generalizadas no grupo de pacientes farmacossensíveis $(p=0,0003)$.

Tabela 2 - Distribuição dos pacientes com epilepsia farmacossensível e farmacorresistente conforme o tipo de epilepsia

\begin{tabular}{cccc}
\hline & $\boldsymbol{n}$ & Epilepsia focal & $\begin{array}{c}\text { Epilepsia } \\
\text { generalizada }\end{array}$ \\
\hline Farmacossensível & 58 & $38(65,52 \%)$ & $20(34,48 \%)$ \\
Farmacorresistente & 56 & $52(92,86 \%)$ & $4(7,14 \%)$ \\
\hline Total & 114 & $90(78,95 \%)$ & $24(21,05 \%)$ \\
\hline
\end{tabular}

$\mathrm{Na}$ distribuição dos pacientes, conforme a quantidade de fármacos em uso (Tabela 3) foi observada o predomínio de politerapia entre os pacientes com epilepsia farmacorresistente $(p<0,0001)$.

Tabela 3 - Distribuição dos pacientes com epilepsia farmacossensível e farmacorresistente quanto à quantidade de fármacos utilizados

\begin{tabular}{cccc}
\hline & $\boldsymbol{n}$ & Monoterapia & Politerapia \\
\hline Farmacossensível & 58 & $34(58,62 \%)$ & $24(41,38 \%)$ \\
Farmacorresistente & 56 & $2(3,57 \%)$ & $54(96,43 \%)$ \\
\hline Total & 114 & $36(31,58 \%)$ & $78(68,42 \%)$ \\
\hline
\end{tabular}


Quanto aos fármacos antiepilépticos, os pacientes farmacorresistentes utilizaram clobazam $(p=0,0003)$ em dose superior aos pacientes farmacossensíveis. Não houve diferença para outros fármacos que estão descritos na Tabela 4.

Tabela 4 - Fármacos antiepilépticos utilizados pelos pacientes

\begin{tabular}{ccccc}
\hline & \multicolumn{2}{c}{ Farmacorresistentes } & \multicolumn{2}{c}{ Farmacossensíveis } \\
\hline & $\mathbf{n}(\%)$ & $\mathbf{m g}$ & $\mathbf{n}(\%)$ & $\mathbf{m g}$ \\
\hline Carbamazepina & $43(76,78 \%)$ & 1118 & $28(48,27 \%)$ & 790 \\
Oxcarbazepina & $15(26,78 \%)$ & 1480 & $8(13,79 \%)$ & 1256 \\
Ácido valpróico & $21(37,50 \%)$ & 1064 & $14(24,14 \%)$ & 1089 \\
Clobazam & $34(60,71 \%)$ & 28 & $11(18,96 \%)$ & 14 \\
Clonazepam & $5(8,92 \%)$ & 2,5 & $4(6,90 \%)$ & 2,31 \\
Nitrazepam & $1(1,78 \%)$ & 20 & 0 & - \\
Topiramato & $19(33,93 \%)$ & 233 & $2(3,45 \%)$ & 200 \\
Lamotrigina & $11(19,64 \%)$ & 339 & $6(10,34 \%)$ & 196 \\
Fenobarbital & $9(16,07 \%)$ & 111 & $4(6,90 \%)$ & 125 \\
Fenitoína & $3(5,36 \%)$ & 200 & $7(12,07 \%)$ & 214 \\
Lacosamida & $3(5,36 \%)$ & 150 & $1(1,72 \%)$ & 200 \\
Levetiracetam & $1(1,78 \%)$ & 3000 & $4(6,90 \%)$ & 1375 \\
Etossuximida & 0 & - & $1(1,72 \%)$ & 500 \\
\hline
\end{tabular}

$\mathrm{n}$ : total de pacientes em uso do medicamento (\% no grupo) 


\subsection{Cronotipo}

As médias do $M E Q$, seus respectivos desvios-padrão e os coeficientes de correlação ponderados pelo LAEP, BDI-II e IDATE estão representados na Tabela 5 .

Tabela 5 - Distribuição das médias, desvios-padrão e coeficientes com valor-p do MEQ entre os grupos controle sem epilepsia, farmacossensível e farmacorresistente

\begin{tabular}{ccccc}
\hline & Média & $\begin{array}{c}\text { Desvio- } \\
\text { padrão }\end{array}$ & Coeficiente & $\mathbf{p}^{*}$ \\
\hline $\begin{array}{c}\text { Controle sem } \\
\text { epilepsia }\end{array}$ & 55,50 & 10,85 & - & - \\
\hline Epilepsia & 50,96 & 9,95 & $-3,330$ & 0,066 \\
\hline Farmacossensível & 51,83 & 10,27 & $-2,97$ & 0,131 \\
Farmacorresistente & 50,05 & 9,61 & $-3,90$ & 0,075 \\
\hline
\end{tabular}

${ }^{*}$ valor $\mathrm{p}<0,05$

Quando se consideram as faixas de cronotipo conforme o resultado do MEQ, observa-se a distribuição representada no Gráfico 1. 
Gráfico 1 - Distribuição dos cronotipos dentro de cada grupo, com o número absoluto de pacientes

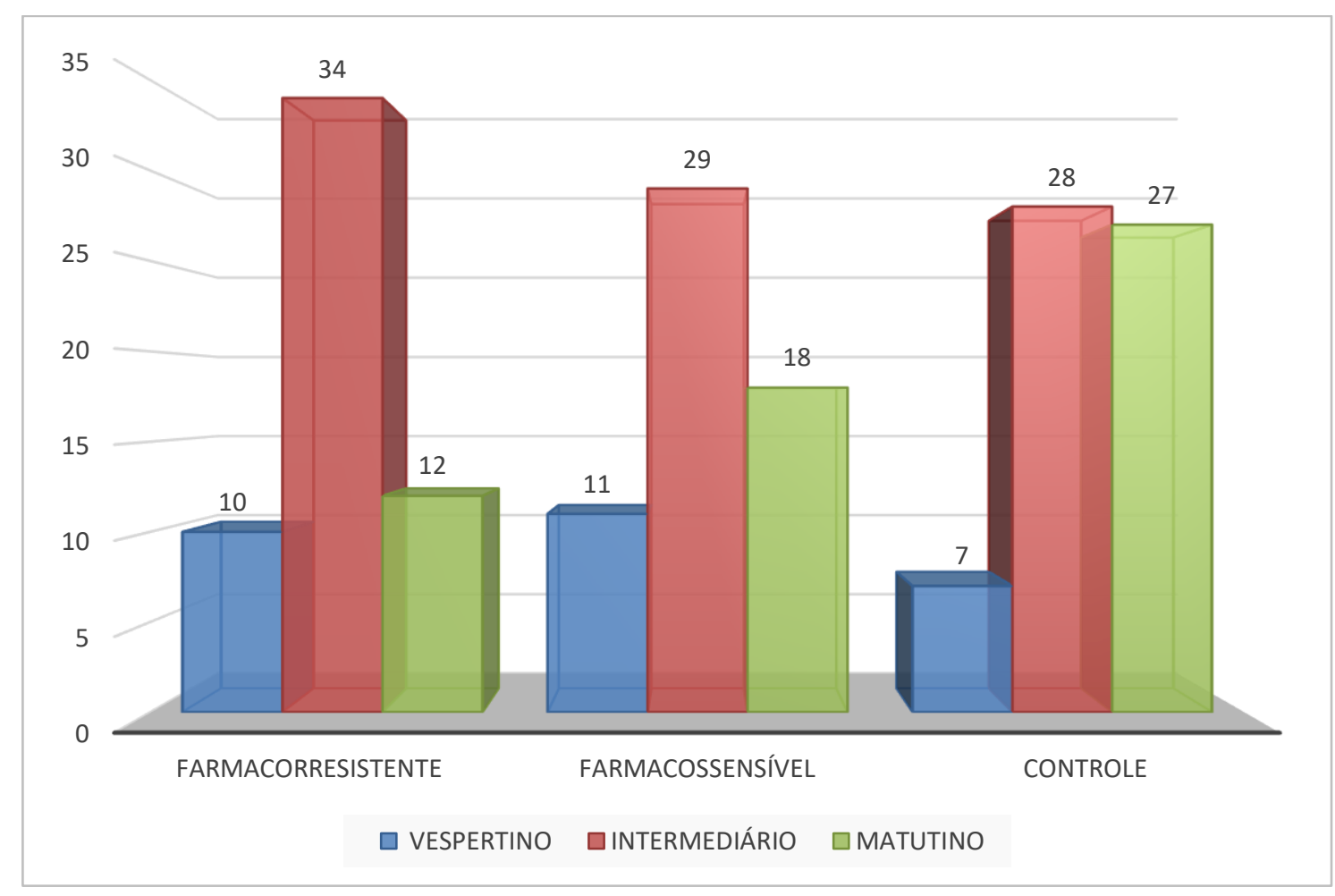

\subsection{Qualidade do Sono}

As médias do PSQI, seus respectivos desvios-padrão e os coeficientes de correlação ponderados pelo LAEP, BDI-II e IDATE estão demonstrados na Tabela 6.

Foi observado que os pacientes farmacorresistentes apresentaram um PSQI 1,53 pontos menor que os controles sem epilepsia e 1,90 pontos menor que os pacientes farmacossensíveis, o que significa uma melhor qualidade de sono. 
Tabela 6 - Distribuição das médias, desvios-padrão e coeficientes com valor-p do PSQI entre os grupos controle sem epilepsia, farmacossensível e farmacorresistente

\begin{tabular}{ccccc}
\hline & Média & $\begin{array}{c}\text { Desvio- } \\
\text { padrão }\end{array}$ & Coeficiente & $\mathbf{p}$ \\
\hline $\begin{array}{c}\text { Controle sem } \\
\text { epilepsia }\end{array}$ & 5,63 & 3,22 & - & - \\
\hline Epilepsia & 6,61 & 3,69 & $-0,360$ & 0,501 \\
\hline Farmacossensível & 7,00 & 3,99 & 0,37 & 0,510 \\
Farmacorresistente & 6,2 & 3,34 & $-1,53$ & $0,016^{*}$ \\
\hline
\end{tabular}

${ }^{*}$ valor $p<0,05$

\subsection{Sonolência Diurna}

As médias do ESS, seus respectivos desvios-padrão e os coeficientes de correlação ponderados pelo LAEP, BDI-II e IDATE estão demonstrados na Tabela 7.

Tabela 7 - Distribuição das médias, desvios-padrão e coeficientes do ESS entre os grupos controle sem epilepsia, farmacossensível e farmacorresistente

\begin{tabular}{ccccc}
\hline & Média & $\begin{array}{c}\text { Desvio- } \\
\text { padrão }\end{array}$ & Coeficiente & $\mathbf{p}$ \\
\hline $\begin{array}{c}\text { Controle sem } \\
\text { epilepsia }\end{array}$ & 7,42 & 4,58 & - & - \\
\hline Epilepsia & 9,57 & 5,81 & 0,379 & 0,671 \\
\hline Farmacossensível & 8,67 & 4,96 & 0,12 & 0,904 \\
Farmacorresistente & 10,50 & 6,49 & 0,80 & 0,459 \\
\hline
\end{tabular}

${ }^{*}$ valor-p $<0,05$ 
5.4. Relação entre os fatores relacionados à epilepsia e o Cronotipo, a Qualidade de Sono e a Sonolência Excessiva Diurna

Como previamente descrito no ítem 5.4.1, os pacientes também foram categorizados pelo tipo de epilepsia (epilepsia focal ou epilepsia generalizada), número de fármacos [monoterapia ou politerapia (dois ou mais fármacos em uso)] e frequência das crises epilépticas.

\subsubsection{Tipo de epilepsia}

As médias e os desvios-padrão para o MEQ, o PSQI e o ESS conforme o tipo de epilepsia estão descritos na Tabela 8. Na análise de contraste entre os grupos - dois a dois e controlados pelo LAEP, BDI-II e IDATE - os pacientes com epilepsia generalizada apresentaram um MEQ 11,99 pontos menor que os pacientes com epilepsia focal $(p=0,0002)$, o que significa uma maior tendência de vespertinidade. Não houve diferença para o PSQI e o ESS. 
Tabela 8 - Distribuição das médias e dos desvios-padrão do MEQ, PSQI e ESS conforme o tipo de epilepsia e diferença corrigida pelo LAEP, BDI-II e IDATE entre os grupos

\begin{tabular}{cccccc}
\hline & & Média & $\begin{array}{c}\text { Desvio- } \\
\text { padrão }\end{array}$ & Diferença & $\mathbf{p}^{*}$ \\
\hline \multirow{2}{*}{ MEQ } & Focal & 52,50 & 9,23 & 11,99 & 0,0002 \\
& Generalizada & 45,30 & 10,70 & & \\
\hline \multirow{2}{*}{ PSQI } & Focal & 6,42 & 3,51 & $-1,28$ & 0,304 \\
& Generalizada & 7,29 & 4,33 & & \\
\hline \multirow{2}{*}{ ESS } & Focal & 9,73 & 5,92 & $-0,50$ & 0,940 \\
& Generalizada & 8,96 & 5,43 & & \\
\hline
\end{tabular}

${ }^{\star} p<0,0005$

\subsubsection{Número de Fármacos Antiepilépticos}

Na distribuição dos pacientes conforme a quantidade de fármacos em uso (Tabela 9), foi observado o predomínio de politerapia entre os pacientes com epilepsia farmacorresistente $(p<0,0001)$.

Tabela 9 - Distribuição dos pacientes com epilepsia farmacossensível e farmacorresistente quanto à quantidade de fármacos utilizados

\begin{tabular}{cccc}
\hline & $\boldsymbol{n}$ & Monoterapia & Politerapia \\
\hline Farmacossensível & 58 & $34(58,62 \%)$ & $24(41,38 \%)$ \\
Farmacorresistente & 56 & $2(3,57 \%)$ & $54(96,43 \%)$ \\
\hline Total & 114 & $36(31,58 \%)$ & $78(68,42 \%)$ \\
\hline
\end{tabular}


As médias e os desvios-padrão para o MEQ, o PSQI e o ESS nos grupos em mono e politerapia estão descritos na Tabela 10. A análise de contraste entre os grupos, dois a dois e controlados pelo LAEP, BDI-II e IDATE, os pacientes em monoterapia apresentam um PSQI 1,75 pontos superior ao grupo em politerapia $(p=0,01)$, o que significa uma pior qualidade de sono na condição de monoterapia.

Tabela 10 - Distribuição das médias e desvios-padrão do MEQ, PSQI e ESS nos grupos de mono e politerapia e diferença entre os grupos.

\begin{tabular}{lccccc}
\hline & & Média & $\begin{array}{c}\text { Desvio- } \\
\text { padrão }\end{array}$ & Diferença & $\mathbf{p}^{*}$ \\
\hline \multirow{2}{*}{ MEQ } & Monoterapia & 49,20 & 11,20 & $-2,90$ & 0,16 \\
& Politerapia & 51,80 & 9,37 & & \\
\hline \multirow{2}{*}{ PSQI } & Monoterapia & 7,56 & 4,21 & 1,75 & $0,01^{*}$ \\
& Politerapia & 6,17 & 3,36 & & \\
\hline \multirow{2}{*}{ ESS } & Monoterapia & 9,22 & 5,21 & $-0,04$ & 0,97 \\
& Politerapia & 9,73 & 6,09 & & \\
\hline
\end{tabular}

${ }^{\star}$ valor $p<0,05$

\subsubsection{Frequência das Crises Epilépticas}

Os 56 pacientes farmacorresistentes foram distribuídos em grupos de frequência de crises epilépticas conforme o relato dos pacientes.

A correlação de Kendall conforme a frequência de crises epilépticas não demonstrou correlação com o MEQ $(-0,13$, IC95\% $-0,38$ a 0,14 p=0,20), 
com o PSQI $(0,11$, IC $95 \%-0,15$ a $0,37 p=0,27)$ ou com o ESS $(-0,04$, IC95\% $0,30$ a $0,22 \mathrm{p}=0,66)$ (Gráfico 2).

Gráfico 2 - Expressão gráfica da correlação entre a frequência de crises e as variáveis MEQ, PSQI e ESS
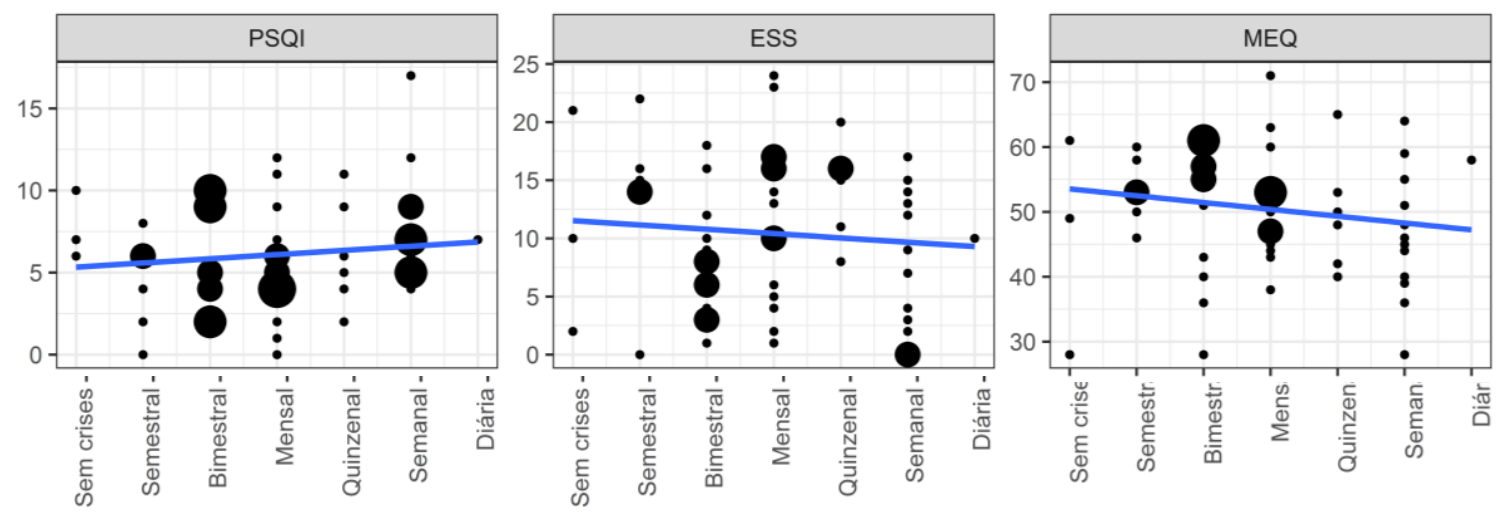

5.5. Relação entre o Cronotipo, a Qualidade de Sono e a Sonolência Excessiva Diurna com a Qualidade de Vida e a Adequação Social

\subsubsection{Qualidade de vida}

Por ser aplicável apenas entre indivíduos com epilepsia, a qualidade de vida faz a comparação apenas entre os grupos de farmacossensibilidade e farmacorresistência. A distribuição dos resultados do QOLIE-31 entre os grupos encontra-se na Tabela 11. 
Tabela 11 - Distribuição dos resultados do QOLIE-31 entre os grupos farmacossensível e farmacorresistente

\begin{tabular}{ccc}
\hline & Média & Desvio-padrão \\
\hline Epilepsia & 59,85 & 20,07 \\
Farmacossensível & 66,78 & 17,59 \\
Farmacorresistente & 52,68 & 20,10 \\
\hline
\end{tabular}

Pacientes com epilepsia farmacorresistentes apresentaram escores menores (6,72 pontos) do que os pacientes farmacossensíveis no QOLIE-31, controlados pelo LAEP, BDI-II e IDATE (IC95\% 2,07 a 11,40; $p=0,005)$.

Pacientes com escores maiores no PSQI apresentaram escores menores no QOLIE-31 ( $p<0,007)$, demonstrando uma correlação marginal negativa para os dois grupos de pacientes, como demonstrado no Gráfico 3.

Gráfico 3 - Correlações entre o Qolie-31 e a qualidade de sono (PSQI), sonolência diurna (ESS) e cronotipo (MEQ)
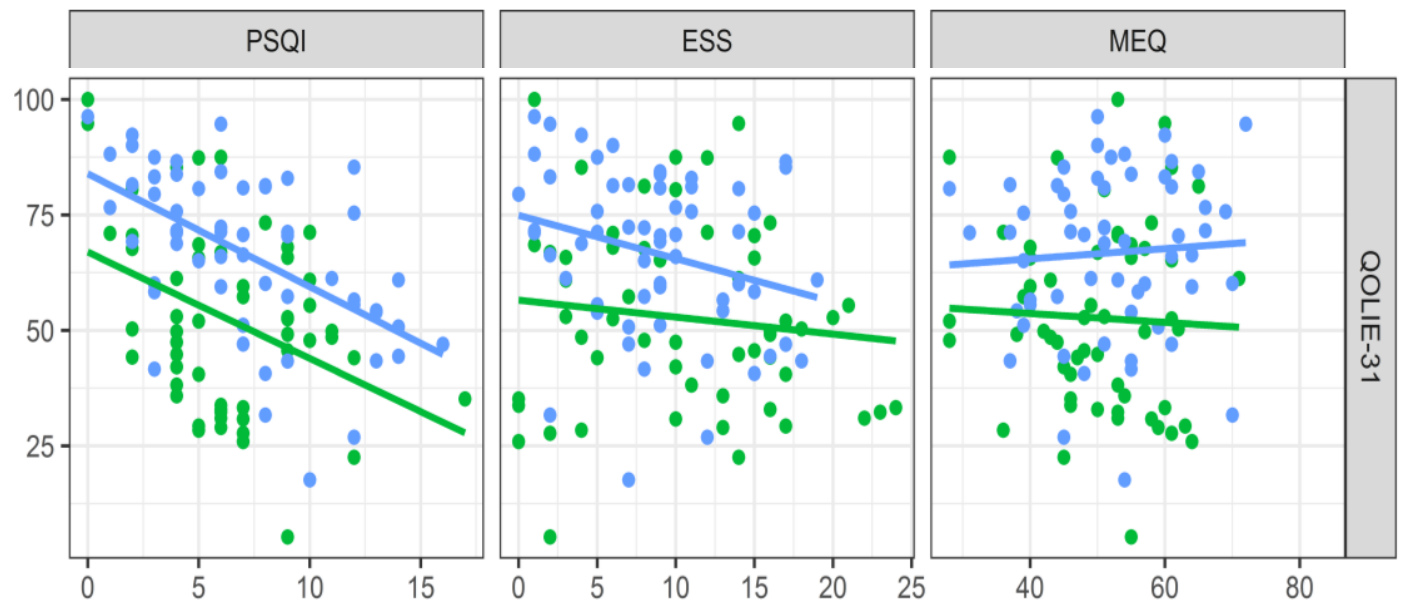

Linha verde: farmacorresistentes; linha azul: farmacossensíveis 


\subsubsection{Adequação social}

A EAS pode ser utilizada nos três grupos: controle sem epilepsia, farmacossensível e farmacorresistente. A distribuição dos resultados do EAS entre os grupos encontra-se na Tabela 12.

Tabela 12 - Distribuição dos resultados do EAS entre os grupos controle sem epilepsia, farmacossensível e farmacorresistente

\begin{tabular}{ccc}
\hline & Média & Desvio-padrão \\
\hline Controle sem epilepsia & 1,89 & 0,55 \\
Epilepsia & 1,96 & 0,50 \\
Farmacossensível & 1,82 & 0,38 \\
Farmacorresistente & 2,11 & 0,57 \\
\hline
\end{tabular}

Não foi observada diferença entre os pacientes farmacorresistentes e o grupo controle quanto aos resultados do EAS. No entanto, os pacientes farmacossensíveis demonstraram um resultado 0,180 pontos menor que 0 controle após controle pelo LAEP, BDI-II e IDATE (IC95\% -0,32 a - 0,04, $\mathrm{p}=0,038)$.

O Gráfico 4 demonstra que há uma interação entre o EAS e resultado do MEQ a depender do grupo, sendo que os farmacorresistentes não diferiram do grupo controle sem epilepsia, mas os farmacossensíveis demonstraram uma interação negativa entre o resultado do MEQ e o EAS $(p=0,011)$. Cada ponto de redução no MEQ corresponde ao aumento de 0,008 
ponto de aumento no EAS, ou seja, a tendência para vespertinidade piora o funcionamento social.

Gráfico 4 - Representação gráfica das correlações entre as escalas MEQ, PSQI e ESS com Qolie-31 e EAS.
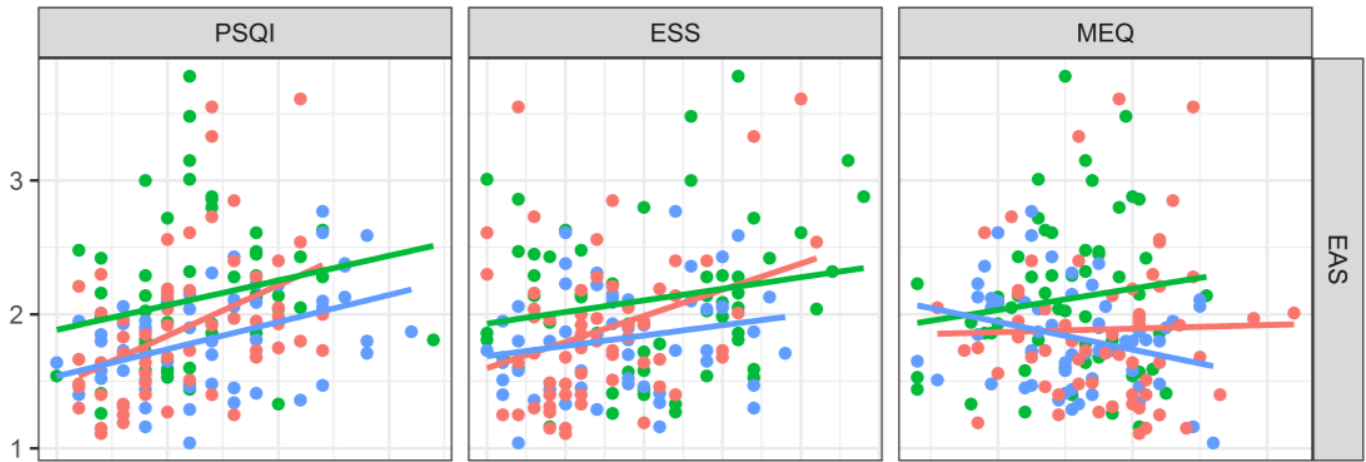

Linha verde: farmacorresistentes; linha azul: farmacossensíveis; linha vermelha: controle sem epilepsia. 


\section{Discussão}

Resumidamente, no presente estudo:

1. Não observamos diferença entre os pacientes com epilepsia farmacorresistente e farmacossensível quanto ao cronotipo e à presença de sonolência diurna;

2. Os indivíduos com epilepsia farmacorresistente apresentaram melhor qualidade de sono em relação aos farmacossensíveis e ao grupo controle sem epilepsia;

3. Os pacientes com epilepsia generalizada tiveram maior tendência à vespertinidade em relação aos indivíduos com epilepsia focal;

4. Pacientes com epilepsia em monoterapia tiveram pior qualidade de sono do que os pacientes em politerapia;

5. A frequência de crises epilépticas não teve influência sobre o cronotipo, a qualidade de sono e a sonolência diurna;

6. Pacientes farmacorresistentes apresentaram pior qualidade de vida. A qualidade de sono teve influência sobre a qualidade de vida e o funcionamento social, mas o mesmo não foi observado com o cronotipo e a sonolência diurna.

Os estudos da relação entre a epilepsia e o sono geralmente comparam pacientes com e sem epilepsia $(20,33,38,77-82)$ ou apenas 
pacientes com epilepsia, por vezes categorizados pelo tipo de epilepsia $(20,29$ 31,33,74-81) No nosso estudo, buscamos demonstrar a relação entre os aspectos do sono e a resposta terapêutica, considerando-se dois grupos farmacorresistentes e farmacossensíveis.

Além disso, nosso estudo ponderou os dados sempre em relação às variáveis de confusão, como perfil de efeitos adversos dos fármacos e a presença dos sintomas ansiosos e depressivos. Embora outros estudos tenham incluído algumas destas variáveis, poucos o fizeram com o mesmo rigor aplicado no nosso estudo, no qual todos foram tratados como fatores confusionais $(20,21,23,33,34,78,79,81,89,91)$.

No nosso estudo, os pacientes farmacorresistentes, farmacossensíveis e controles sem epilepsia não apresentaram diferença quanto à sonolência diurna. Estes resultados estão em concordância com outros autores, nos quais pacientes com diferentes tipos de epilepsia não apresentaram sonolência excessiva durante o dia quando comparados aos controles $(21,22,33,38,59,80-82)$. Poucos autores encontraram sonolência diurna nos pacientes com epilepsia (77-79). No único estudo com características similares às nossas, García-Morales et al. (15) avaliaram pacientes com epilepsia focal farmacorresistente e farmacossensível, demonstrando uma maior frequência de insônia e sonolência diurna nos farmacorresistentes. A nossa hipótese para esta discrepância é a não covariação para efeitos adversos dos fármacos que podem ter um impacto importante nos aspectos relacionados ao sono, em especial na sonolência. 
Assim como a análise da sonolência diurna, o estudo do cronotipo, também apresenta resultados controversos. A análise de pacientes com epilepsia do lobo temporal, epilepsia do lobo frontal e epilepsia mioclônica juvenil, demonstrou uma tendência à matutinidade nos pacientes com epilepsia, a despeito do tipo de epilepsia (24). Corroborando parcialmente o estudo anterior, Manni et al. (20), em um estudo com 60 pacientes com epilepsia focal sem diferenciação quanto à farmacorresposividade, demonstraram uma tendência à matutinidade nos pacientes com epilepsia focal. Por outro lado, entretanto, a análise do valor médio do MEQ destes estudos $(20,24)$ demonstrou que os pacientes apresentavam o cronotipo intermediário, como foi observado no nosso grupo. Unterberger et al. (21), utilizando uma escala distinta - Munich Chronotype Questionnaire - analisaram 100 pacientes com epilepsia focal, 100 pacientes com epilepsia generalizada e 100 indivíduos saudáveis, não encontraram qualquer tipo de diferença quanto ao cronotipo.

$\mathrm{Na}$ análise dos nossos grupos em relação ao tipo de epilepsia, quantidade de fármacos utilizados e à frequência de crises observamos maior tendência à vespertinidade entre os pacientes com epilepsia generalizada e pior qualidade de sono entre os pacientes sob monoterapia (que será discutido adiante). A frequência de crises não demonstrou correlação com o cronotipo, a qualidade de sono e a sonolência diurna. Essa tendência à vespertinidade na epilepsia generalizada é frequentemente descrita $(22,23,91)$. A tendência à vespertinidade nos pacientes com epilepsia generalizada pode ser explicada pela presença dos pacientes com epilepsia mioclônica juvenil, cuja vespertinidade foi bem delineada por Pung \& Schmitz (23). Esta foi a síndrome 
epiléptica generalizada predominante no nosso estudo e nos anteriores $(22,23,91)$.

Como discutido anteriormente por Hofstra et al. (24), as diferenças de cronotipo entre os estudos não são facilmente explicáveis dado a heterogeneidade dos tipos de epilepsia, dos questionários aplicados e dados demográficos, como a idade.

Em relação à qualidade do sono, os pacientes com epilepsia farmacorresistente apresentaram, em uma análise subjetiva baseada na percepção do indivíduo, melhor qualidade do sono do que os pacientes farmacossensíveis e controles saudáveis. Este foi um ponto de maior discordância entre o nosso estudo e os estudos anteriores, onde se descreve um predomínio de pior qualidade de sono entre os pacientes com epilepsia senso lato $(33,77,79,80,82)$ ou nos pacientes farmacorresistentes (59). A ausência de diferença na qualidade do sono entre os indivíduos com epilepsia e sem epilepsia também é descrita $(20,21,78)$. Choi et al. (22) não observaram diferença no resultado do PSQI ao compararem 127 pacientes com epilepsia focal, 33 indivíduos com epilepsia generalizada e 100 controles sem epilepsia. Entretanto, o resultado de melhor qualidade de sono nos pacientes com epilepsia farmacorresistente não é esperado. Um dos fatores que pode explicar este dado é o uso noturno de benzoadiazepínicos, em especial clobazam e clonazepam, em todos os pacientes com epilepsia farmacorresistente. Os benzodiazepínicos, que são medicações hipnóticas e ansiolíticas, não são reconhecidas pelos pacientes como medicação para induzir o sono (um dos itens do PSQI) e podem impactar positivamente cinco dos sete fatores deste questionário utilizado para 
avaliar a qualidade do sono (ex., duração do sono, redução da latência para dormir, aumentar a eficiência, atenuar a percepção de distúrbios de sono, melhorar a percepção de distúrbios do sono). Este achado de melhor qualidade de sono em pacientes farmacorresistentes também poderia explicar a melhor qualidade de sono nos pacientes em politerapia em relação aos pacientes em monoterapia.

Sobre a qualidade de vida, observou-se uma relação com a qualidade do sono, mas sem a associação com o cronotipo e a sonolência diurna. Pacientes com epilepsia farmacorresistente apresentaram pior qualidade de vida do que pacientes com epilepsia farmacossensível. Embora a epilepsia per se possa ser um fator determinante de pior qualidade de vida $(46,47)$, a presença de farmacorresistência foi relevante no nosso estudo. Deve-se enfatizar que outros autores não observaram relação entre a maior frequência de crises e a pior qualidade de vida $(46,92-94)$. Entretanto, o conceito de farmacorresistência é mais abrangente do que somente a maior frequência das crises epilépticas.

A presença de uma correlação entre qualidade de sono e qualidade de vida é recorrente em estudos com tipos diferentes de pacientes $(15,19,21,32$ 35,61,84,85,87-89). O instrumento utilizado neste estudo é direcionado à qualidade de vida em epilepsia e acreditamos que isto seja um diferencial visto que estudos que utilizaram instrumentos genéricos (ex. SF-36) apresentam resultados discrepantes $(33,36,38)$. Nos estudos com QOLIE-10, 31 e 89 , os resultados quanto à qualidade de sono e qualidade de vida são similares aos nossos $(32,34,35,37)$. 
O estudo do funcionamento social, através da escala de adequação social, nos pacientes com epilepsia foi realizado anteriormente pelo nosso grupo para comorbidades cognitivas e psiquiátricas. O estudo de Gois et al. (40) demonstrou que pacientes com epilepsia do lobo temporal, determinada por esclerose de hipocampo, têm pior funcionamento social do que controles saudáveis. As variáveis relacionadas à pior adequação social foram os déficits cognitivos e a farmacorresistência, quando covariado para depressão e ansiedade. O estudo de Moschetta \& Valente (39), em pacientes com epilepsia mioclônica juvenil, demonstrou que a impulsividade e a presença de crises mioclônicas tônico clônicas são os fatores determinantes de pior funcionamento social. Este é o primeiro estudo avaliando a relação entre a qualidade de sono, o cronotipo e a sonolência diurna e o funcionamento social. Os pacientes farmacossensíveis com tendência à vespertinidade apresentaram pior adequação social. Como neste grupo há uma elevada proporção de epilepsia generalizada, principalmente epilepsia mioclônica juvenil, os nossos achados corroboram os estudos anteriores quanto a relevância de múltiplos fatores no ajuste social que não são restritos apenas à frequência das crises epilépticas. Portanto, nos pacientes com epilepsia, o funcionamento social é determinado por fatores relacionados à epilepsia e às suas comorbidades.

Assim, os achados sugerem que a relação entre o sono e a epilepsia está mais intimamente ligada ao tipo de epilepsia, independente da resposta terapêutica ou da frequência de crises epilépticas. Além do mais, esta relação está ligada ao cronotipo, com tendência de vespertinidade nos pacientes com epilepsia generalizada em relação aos pacientes com epilepsia focal. A 
qualidade de vida e o funcionamento social também estão concatenadas à qualidade do sono de forma direta, tanto em pacientes farmacorresistentes quanto farmacossensíveis. 


\section{Conclusão}

1. Pacientes farmacorresistentes não apresentaram alterações do cronotipo e maior sonolência diurna, mas apresentaram melhor qualidade de sono;

2. Pacientes com epilepsia generalizada apresentaram tendência à vespertinidade, pacientes em monoterapia apresentaram pior qualidade de sono e a frequência de crises epilépticas não se correlacionou com o cronotipo, a qualidade do sono e a sonolência diurna;

3. A pior qualidade de sono foi um fator preditor de pior qualidade de vida e de adequação social nos pacientes com epilepsia. 


\section{Bibliografia}

1. Foldvary-Schaefer N, Grigg-Damberger M. Sleep and epilepsy: what we know, don't know, and need to know. J Clin Neurophysiol . fevereiro de $2006 ; 23(1): 4-20$.

2. Dieter J. The epilepsies. In: Vinken P, Bruyn G, organizadores. Handbook of Clinical Neurology. Amsterdam: North-Holland Publishing Company; 1974. p. 457-90.

3. Dieter J. The grand mal epilepsies and the sleeping-waking cycle. Epilepsia. 1962;3(69-109).

4. Scheffer IE, Berkovic S, Capovilla G, Connolly MB, French J, Guilhoto L, et al. ILAE classification of the epilepsies: Position paper of the ILAE Commission for Classification and Terminology. Epilepsia. $2017 ; 58(4): 512-21$.

5. Janz D. Epilepsy with grand mal on awakening and sleep-waking cycle. Clin Neurophysiol . setembro de 2000;111 Suppl:S103-10.

6. Adachi N, Alarcon G, Binnie CD, Elwes RD, Polkey CE, Reynolds EH. Predictive value of interictal epileptiform discharges during non-REM sleep on scalp EEG recordings for the lateralization of epileptogenesis. Epilepsia . junho de 1998;39(6):628-32. 
7. Clemens Z, Janszky J, Szucs A, Békésy M, Clemens B, Halász P. Interictal epileptic spiking during sleep and wakefulness in mesial temporal lobe epilepsy: a comparative study of scalp and foramen ovale electrodes. Epilepsia . fevereiro de 2003;44(2):186-92.

8. Sammaritano M, Gigli GL, Gotman J. Interictal spiking during wakefulness and sleep and the localization of foci in temporal lobe epilepsy. Neurology . fevereiro de 1991;41(2 ( Pt 1)):290-7.

9. Bazil CW, Walczak TS. Effects of sleep and sleep stage on epileptic and nonepileptic seizures. Epilepsia . janeiro de 1997;38(1):56-62.

10. Herman ST, Walczak TS, Bazil CW. Distribution of partial seizures during the sleep--wake cycle: differences by seizure onset site. Neurology. 2001;56(11):1453-9.

11. Manni R, Terzaghi M, Arbasino C, Sartori I, Galimberti CA, Tartara A. Obstructive sleep apnea in a clinical series of adult epilepsy patients: frequency and features of the comorbidity. Epilepsia . junho de 2003;44(6):836-40.

12. Malow BA, Levy K, Maturen K, Bowes R. Obstructive sleep apnea is common in medically refractory epilepsy patients. Neurology . 10 de outubro de 2000;55(7):1002-7.

13. Hollinger P, Khatami R, Gugger M, Hess CW, Bassetti CL. Epilepsy and obstructive sleep apnea. Eur Neurol . 2006;55(2):74-9.

14. Vendrame M, Auerbach S, Loddenkemper T, Kothare S, Montouris G. 
Effect of continuous positive airway pressure treatment on seizure control in patients with obstructive sleep apnea and epilepsy. Epilepsia . novembro de 2011;52(11):e168-71.

15. García-morales I, Gil-nagel A, Rosendo J De, Torres-falcón A. Alteraciones del sueño y calidad de vida en la epilepsia parcial refractaria : resultados del estudio SLEEP. 2014;58(4):152-60.

16. Yu-Dan L, Zan W, Ma D-H, Meng H-M, Cui L. Association between epileptiform discharges and the sleep cycle in 200 epileptic patients. Int J Neurosci . março de 2013;123(3):196-203.

17. Krishnan P, Sinha S, Taly AB, Ramachandraiah CT, Rao S, Satishchandra P. Altered polysomnographic profile in juvenile myoclonic epilepsy. Epilepsy Res . março de 2014;108(3):459-67.

18. Giorelli AS, Passos P, Carnaval T, Gomes M da M. Excessive daytime sleepiness and epilepsy: a systematic review. Epilepsy Res Treat . 2013;2013:629469.

19. Chen N-C, Tsai M-H, Chang C-C, Lu C-H, Chang W-N, Lai S-L, et al. Sleep quality and daytime sleepiness in patients with epilepsy. Acta Neurol Taiwan . dezembro de 2011;20(4):249-56.

20. Manni R, Cremascoli R, De Icco R, Terzaghi M. Chronotype in patients with epilepsy: A controlled study in 60 subjects with late-onset focal epilepsy. Epilepsy Behav . 2015;50:1-6.

21. Unterberger I, Gabelia D, Prieschl M, Chea K, Hofer M, Högl B, et al. 
Sleep disorders and circadian rhythm in epilepsy revisited: A prospective controlled study. Sleep Med . 2015;16(2):237-42.

22. Choi SJ, Joo EY, Hong SB. Sleep-wake pattern, chronotype and seizures in patients with epilepsy. Epilepsy Res . 2016;120:19-24.

23. Pung T, Schmitz B. Circadian rhythm and personality profile in juvenile myoclonic epilepsy. Epilepsia. 2006;47(SUPPL. 2):111-4.

24. Hofstra WA, Gordijn MCM, Hemert-van JC Van, Poel D, Palen J Van Der, Weerd AW De. Chronotype and subjective sleep parameters in epilepsy patients: a large questionnaire study. Chronobiol Int. 2010;27(6):1271-86.

25. Batista BHB, Nunes ML. Evaluation of sleep habits in children with epilepsy. Epilepsy Behav. 2007;11(1):60-4.

26. Kuhlman SJ, Mackey SR, Duffy JF. Biological Rhythms Workshop I: introduction to chronobiology. Cold Spring Harb Symp Quant Biol . $2007 ; 72: 1-6$.

27. Wright KP, McHill AW, Birks BR, Griffin BR, Rusterholz T, Chinoy ED. Entrainment of the human circadian clock to the natural light-dark cycle. Curr Biol . 19 de agosto de 2013;23(16):1554-8.

28. Quigg M, Straume M, Smith T, Menaker M, Bertram EH. Seizures induce phase shifts of rat circadian rhythms. Brain Res. 2001;913(2):165-9.

29. Linkowski P, Mendlewicz J, Kerkhofs M, Leclercq R, Golstein J, Brasseur M, et al. 24-hour profiles of adrenocorticotropin, cortisol, and growth 
hormone in major depressive illness: effect of antidepressant treatment. $\mathrm{J}$ Clin Endocrinol Metab . julho de 1987;65(1):141-52.

30. Horne JA, Ostberg O. A self-assessment questionnaire to determine morningness-eveningness in human circadian rhythms. Int $\mathrm{J}$ Chronobiol . 1976;4(2):97-110.

31. Quigg M, Straume M, Smith T, Menaker M, Bertram EH. Seizures induce phase shifts of rat circadian rhythms. Brain Res . 21 de setembro de 2001;913(2):165-9.

32. Alanis-Guevara I, Peña E, Corona T, López-Ayala T, López-Meza E, López-Gómez M. Sleep disturbances, socioeconomic status, and seizure control as main predictors of quality of life in epilepsy. Epilepsy Behav. 2005;7(3):481-5.

33. de Weerd A, de Haas S, Otte A, Trenité DK-N, van Erp G, Cohen A, et al. Subjective sleep disturbance in patients with partial epilepsy: a questionnaire-based study on prevalence and impact on quality of life. Epilepsia . 2004;45(11):1397-404.

34. Piperidou C, Karlovasitou A, Triantafyllou N, Terzoudi A, Constantinidis T, Vadikolias K, et al. Influence of sleep disturbance on quality of life of patients with epilepsy. Seizure. 2008;17(7):588-94.

35. Kwan P, Yu E, Leung H, Leon T, Mychaskiw MA. Association of subjective anxiety, depression, and sleep disturbance with quality-of-life ratings in adults with epilepsy. Epilepsia. 2009;50(5):1059-66. 
36. Hermann BP, Seidenberg M, Bell BD, Woodard A, Rutecki P, Sheth R. Comorbid psychiatric symptoms in temporal lobe epilepsy: association with chronicity of epilepsy and impact on quality of life. Epilepsy Behav. 2000;1(3):184-90.

37. Johnson E, Jones J, Seidenberg M, Hermann B. The relative impact of axiety, depression and clinical seizure features on health-related quality of life in epilepsy. Epilepsia. 2004;45(5):544-50.

38. Jacoby A, Snape D, Lane S, Baker GA. Self-reported anxiety and sleep problems in people with epilepsy and their association with quality of life. Epilepsy Behav . 2015;43:149-58.

39. Moschetta S, Valente KD. Impulsivity and seizure frequency, but not cognitive deficits, impact social adjustment in patients with juvenile myoclonic epilepsy. Epilepsia. 2013;54(5):866-70.

40. Gois J, Valente K, Vicentiis S, Moschetta S, Kuczynski E, Fiore L, et al. Assessment of psychosocial adjustment in patients with temporal lobe epilepsy using a standard measure. Epilepsy Behav . 2011;20(1):89-94.

41. Gutter T, Brouwer OF, de Weerd AW. Subjective sleep disturbances in children with partial epilepsy and their effects on quality of life. Epilepsy Behav . 2013;28(3):481-8.

42. Placidi F, Diomedi M, Scalise A, Marciani MG, Romigi A, Gigli GL. Effect of anticonvulsants on nocturnal sleep in epilepsy. Neurology . 2000;54(5 Suppl 1):S25-32. 
43. Legros B, Bazil CW. Effects of antiepileptic drugs on sleep architecture: a pilot study. Sleep Med . janeiro de 2003;4(1):51-5.

44. Salinsky MC, Oken BS, Binder LM. Assessment of drowsiness in epilepsy patients receiving chronic antiepileptic drug therapy. Epilepsia. 1996;37(2):181-7.

45. Valente KDR, Busatto Filho G. Depression and temporal lobe epilepsy represent an epiphenomenon sharing similar neural networks: clinical and brain structural evidences. Arq Neuropsiquiatr . 2013;71(3):183-90.

46. Gilliam F. Optimizing health outcomes in active epilepsy. Neurology. 2002;58(8 Suppl 5):S9-20.

47. Luoni C, Bisulli F, Canevini MP, De Sarro G, Fattore C, Galimberti CA, et al. Determinants of health-related quality of life in pharmacoresistant epilepsy: results from a large multicenter study of consecutively enrolled patients using validated quantitative assessments. Epilepsia . dezembro de 2011;52(12):2181-91.

48. Elsharkawy AE, Thorbecke R, Ebner A, May TW. Determinants of quality of life in patients with refractory focal epilepsy who were not eligible for surgery or who rejected surgery. Epilepsy Behav . 2012;24(2):249-55.

49. Pompili M, Serafini G, Innamorati M, Montebovi F, Lamis D a, Milelli M, et al. Factors associated with hopelessness in epileptic patients. World $\mathrm{J}$ psychiatry. 2014;4(4):141-9.

50. Schneider-Von Podewils F, Gasse C, Geithner J, Wang ZI, Bombach P, 
Berneiser $\mathrm{J}$, et al. Clinical predictors of the long-term social outcome and quality of life in juvenile myoclonic epilepsy: 20-65 years of follow-up. Epilepsia. 2014;55(2):322-30.

51. Kwan P, Arzimanoglou A, Berg AT, Brodie MJ, Hauser WA, Mathern G, et al. Definition of drug resistant epilepsy: Consensus proposal by the ad hoc Task Force of the ILAE Commission on Therapeutic Strategies. Epilepsia. 2010;51(6):1069-77.

52. Kobau R, Zahran H, Thurman DJ, Zack MM, Henry TR, Schachter SC PP. Epilepsy surveillance among adults--19 States, Behavioral Risk Factor Surveillance System, 2005. MMWR Surveill Summ. 2008;57(6):120.

53. Begley CE, Famulari M, Annegers JF, Lairson DR, Reynolds TF, Coan S, Dubinsky S, Newmark ME, Leibson C, So EL RW. The cost of epilepsy in the United States: an estimate from population-based clinical and survey data. Epilepsia. 2000;41(3):342-51.

54. Sperling MR, Barshow S, Nei M A-PA. A reappraisal of mortality after epilepsy surgery. Neurology. 2016;86(21):1938-44.

55. Pinikahana J, Dono J. Age and gender differences in initial symptoms and precipitant factors of epileptic seizures: an Australian study. Epilepsy Behav . outubro de 2009;16(2):231-9.

56. Balamurugan E, Aggarwal M, Lamba A, Dang N, Tripathi M. Perceived trigger factors of seizures in persons with epilepsy. Seizure . novembro de 
2013;22(9):743-7.

57. Nakken KO, Solaas MH, Kjeldsen MJ, Friis ML, Pellock JM, Corey LA. Which seizure-precipitating factors do patients with epilepsy most frequently report? Epilepsy Behav . fevereiro de 2005;6(1):85-9.

58. Vieira Scarlatelli-Lima A, Sukys-Claudino L, Watanabe N, Guarnieri R, Walz R, Lin K. How do people with drug-resistant mesial temporal lobe epilepsy sleep? A clinical and video-EEG with EOG and submental EMG for sleep staging study. eNeurologicalSci. 2016;4:34-41.

59. Ismayilova V, Demir AU, Tezer FI. Subjective sleep disturbance in epilepsy patients at an outpatient clinic: A questionnaire-based study on prevalence. Epilepsy Res . 2015;115:119-25.

60. Chen Y-Y, Huang S, Wu W-Y, Liu C-R, Yang X-Y, Zhao H-T, et al. Associated and predictive factors of quality of life in patients with temporal lobe epilepsy. Epilepsy Behav. 2018;86:85-90.

61. Fisher RS, Acevedo C, Arzimanoglou A, Bogacz A, Cross JH, Elger CE, et al. ILAE Official Report: A practical clinical definition of epilepsy. Epilepsia. 2014;55(4):475-82.

62. Benedito-Silva AA, Menna-Barreto L, Marques N, Tenreiro S. A selfassessment questionnaire for the determination of morningnesseveningness types in Brazil. Prog Clin Biol Res . 1990;341B:89-98.

63. Buysse DJ, Reynolds CF, Monk TH, Berman SR, Kupfer DJ. The Pittsburgh Sleep Quality Index: a new instrument for psychiatric practice 
and research. Psychiatry Res . maio de 1989;28(2):193-213.

64. Bertolazi AN, Fagondes SC, Hoff LS, Dartora EG, Miozzo IC da S, de Barba MEF, et al. Validation of the Brazilian Portuguese version of the Pittsburgh Sleep Quality Index. Sleep Med . janeiro de 2011;12(1):70-5.

65. Johns MW. A new method for measuring daytime sleepiness: the Epworth sleepiness scale. Sleep . dezembro de 1991;14(6):540-5.

66. Bertolazi AN, Fagondes SC, Hoff LS, Pedro VD, Menna Barreto SS, Johns MW. Portuguese-language version of the Epworth sleepiness scale: validation for use in Brazil. J Bras Pneumol . setembro de 2009;35(9):877-83.

67. Cramer JA, Perrine K, Devinsky O, Bryant-Comstock L, Meador K, Hermann B. Development and cross-cultural translations of a 31-item quality of life in epilepsy inventory. Epilepsia . janeiro de 1998;39(1):81-8.

68. da Silva TI, Ciconelli RM, Alonso NB, Azevedo AM, Westphal-Guitti AC, Pascalicchio TF, et al. Validity and reliability of the Portuguese version of the quality of life in epilepsy inventory (QOLIE-31) for Brazil. Epilepsy Behav . março de 2007;10(2):234-41.

69. Weissman MM, Bothwell S. Assessment of social adjustment by patient self-report. Arch Gen Psychiatry . setembro de 1976;33(9):1111-5.

70. Gorenstein C, Moreno RA, Bernik MA, Carvalho SC, Nicastri S, Cordás T, et al. Validation of the Portuguese version of the Social Adjustment Scale on Brazilian samples. J Affect Disord . maio de 2002;69(1-3):167-75. 
71. Baker G, Jacoby A, Francis P, Chadwick D. The Liverpool adverse drug events profile. Epilepsia1. 995;36:S59.

72. Martins HH, Alonso NB, Vidal-Dourado M, Carbonel TD, de Araújo Filho GM, Caboclo LO, et al. Are adverse effects of antiepileptic drugs different in symptomatic partial and idiopathic generalized epilepsies? The Portuguese-Brazilian validation of the Liverpool Adverse Events Profile. Epilepsy Behav . novembro de 2011;22(3):511-7.

73. Beck A, Steer R, Brown G. Beck Depression Inventory-Second Edition. San Antonio, TX: Psychological Corporation; 1996.

74. Gorenstein C, Andrade L. Validation of a Portuguese version of the Beck Depression Inventory and the State-Trait Anxiety Inventory in Brazilian subjects. Brazilian J Med Biol Res $=$ Rev Bras Pesqui medicas e Biol . abril de 1996;29(4):453-7.

75. Spielberger C, Gorsuch R, Lushene R, Vag P, Jacobs G. Manual for the State-Trait Anxiety Inventory. Palo Alto, CA: Consulting Psychologists Press; 1983.

76. Gorenstein C, Andrade L. Validation of a Portuguese version of the Beck Depression Inventory and the State-Trait Anxiety Inventory in Brazilian subjects. Brazilian J Med Biol Res $=$ Rev Bras Pesqui medicas e Biol. abril de 1996;29(4):453-7.

77. Im H-J, Park S-H, Baek S-H, Chu MK, Yang KI, Kim W-J, et al. Associations of impaired sleep quality, insomnia, and sleepiness with 
epilepsy: A questionnaire-based case-control study. Epilepsy Behav. 2016;57:55-9.

78. Pizzatto R, Lin K, Watanabe N, Campiolo G, Alice M, Bicalho H, et al. Excessive sleepiness and sleep patterns in patients with epilepsy: A case-control study. Epilepsy Behav. 2013;29:63-6.

79. Yazdi Z, Sadeghniiat-Haghighi K, Naimian S, Zohal MA, Ghaniri M. Prevalence of Sleep Disorders and their Effects on Sleep Quality in Epileptic Patients A B S T R A C T. Vol. 4. 2013.

80. Shen $\mathrm{Y}$, Zhang M, Wang $\mathrm{Y}$, Wang L, Xu X, Xiao G. Subjective sleep disturbance in Chinese adults with epilepsy: Associations with a ff ective symptoms. Epilepsy Res . 2017;135(210):150-7.

81. Khatami R, Zutter D, Siegel A, Mathis J, Donati F, Bassetti CL. Sleepwake habits and disorders in a series of 100 adult epilepsy patients-A prospective study. Seizure. 2006;15(5):299-306.

82. Moser D, Pablik E, Aull-Watschinger S, Pataraia E, Wöber C, Seidel S. Depressive symptoms predict the quality of sleep in patients with partial epilepsy - A combined retrospective and prospective study. Epilepsy Behav. 2015;47:104-10.

83. Vendrame M, Yang B, Jackson S, Auerbach SH. Insomnia and epilepsy: a questionnaire-based study. J Clin Sleep Med . 1 de fevereiro de 2013;9(2):141-6.

84. Camara-Lemarroy CR, Hoyos M, Ibarra-Yruegas BE, Díaz-Torres MA, De 
León R. Affective symptoms and determinants of health-related quality of life in Mexican people with epilepsy.

85. Camilo A, Espinosa J, Ramírez Salazar S, Rincón Rodríguez C, Ernesto F, Mejía S. Factors associated with quality of life in a low-income population with epilepsy. Epilepsy Res. 2016;127:168-74.

86. Yi P-L, Chen Y-J, Lin C-T, Chang F-C, P.-L. Y, Y.-J. C, et al. Occurrence of epilepsy at different zeitgeber times alters sleep homeostasis differently in rats. Sleep. 2012;35(12):1651-65.

87. Chen H-F, Tsai Y-F, Hsi M-S, Chen J-C. Factors affecting quality of life in adults with epilepsy in Taiwan: A cross-sectional, correlational study. 2016;

88. Giorelli AS, Schenkel De Moura G, Neves L, Venturi M, Pontes IM, Valois A, et al. Excessive daytime sleepiness in patients with epilepsy: A subjective evaluation. Epilepsy Behav. 2011;21:449-52.

89. Quigg M, Gharai S, Ruland J, Schroeder C, Hodges M, Ingersoll KS, et al. Insomnia in epilepsy is associated with continuing seizures and worse quality of life. Epilepsy Res . 2016;122:91-6.

90. Gómez-Arias B, Crail-Meléndez D, López-Zapata R, Martínez-Juárez IE. Severity of anxiety and depression are related to a higher perception of adverse effects of antiepileptic drugs. Seizure. 2012;21(8):588-94.

91. Kendis H, Baron K, Schuele SU, Patel B, Attarian H. Chronotypes in patients with epilepsy: Does the type of epilepsy make a difference? 
Behav Neurol. 2015;2015.

92. Viteva EI. Seizure frequency and severity: How really important are they for the quality of life of patients with refractory epilepsy. Ann Indian Acad Neurol . janeiro de 2014;17(1):35-42.

93. Elsharkawy AE, May T, Thorbecke R, Koch-Stoecker S, Villagran A, Urak L, et al. Long-term outcome and determinants of quality of life after temporal lobe epilepsy surgery in adults. Epilepsy Res . outubro de 2009;86(2-3):191-9.

94. Tracy JI, Dechant V, Sperling MR, Cho R, Glosser D. The association of mood with quality of life ratings in epilepsy. Neurology . 3 de abril de 2007;68(14):1101-7. 


\section{Anexos}

Anexo 1 - Caracterização dos pacientes farmacorresistentes quanto à etiologia

\begin{tabular}{|c|c|c|}
\hline Paciente & Etiologia da epilepsia & Área acometida \\
\hline 1 & Displasia focal & Frontal \\
\hline 2 & Esclerose mesial temporal (EMT) & Temporal \\
\hline 3 & EMT & Temporal \\
\hline 4 & Heterotopia & Temporal \\
\hline 5 & EMT & Temporal \\
\hline 6 & Heterotopia & Temporal \\
\hline 7 & Focal indeterminada & Frontotemporal \\
\hline 8 & EMT & Temporal \\
\hline 9 & Displasia cortical & Frontal \\
\hline 10 & Focal indeterminada & Temporal \\
\hline 11 & EMT & Temporal \\
\hline 12 & Heterotopia & Frontal \\
\hline 13 & EMT & Temporal \\
\hline 14 & Focal indeterminada & Frontal \\
\hline 15 & EMT & Bilateral \\
\hline 16 & Focal indeterminado & Frontal \\
\hline 17 & Heterotopia & Frontal \\
\hline 18 & Focal indeterminada & Frontal \\
\hline 19 & Esquizencefalia & Frontal \\
\hline 20 & Focal indeterminada & Frontal \\
\hline 21 & Focal indeterminada & Temporal \\
\hline 22 & Gliose por traumatismo crânio-encefálico & Frontal \\
\hline 23 & Neoplasia & Temporal \\
\hline 24 & EMT & Temporal \\
\hline 25 & EMT & Temporal \\
\hline 26 & Indeterminada & Multifocal \\
\hline 27 & Temporal & Temporal \\
\hline 28 & EMT & Temporal \\
\hline
\end{tabular}


Anexo 1 - Caracterização dos pacientes farmacorresistentes quanto à etiologia (conclusão)

\begin{tabular}{|c|c|c|}
\hline 29 & Cavernoma & Temporal \\
\hline 30 & EMT & Temporal \\
\hline 31 & Focal & Temporal \\
\hline 32 & Epilepsia com crise tônico-clônica apenas & Generalizada \\
\hline 33 & Focal indeterminada & Frontal \\
\hline 34 & Focal indeterminada & Temporal \\
\hline 35 & Focal indeterminada & Frontal \\
\hline 36 & Glioma & Temporal \\
\hline 37 & Mal desenvolvimento cortical & Frontotempora \\
\hline 38 & Heterotopia & Frontal \\
\hline 39 & Gliose por traumatismo crânio-encefálico & Frontal \\
\hline 40 & Polimicrogiria & Frontal \\
\hline 41 & EMT & Temporal \\
\hline 42 & EMT & Temporal \\
\hline 43 & Epilepsia mioclônica juvenil (EMJ) & Generalizada \\
\hline 44 & Heterotopia & Temporal \\
\hline 45 & EMJ & Generalizada \\
\hline 46 & Esquizencefalia & Frontal \\
\hline 47 & Gliose & Frontal \\
\hline 48 & Focal indeterminado & Frontal \\
\hline 49 & EMT & Temporal \\
\hline 50 & EMT & Temporal \\
\hline 51 & EMT & Temporal \\
\hline 52 & EMT & Temporal \\
\hline 53 & EMT & Temporal \\
\hline 54 & Focal indeterminado & Temporal \\
\hline 55 & Heterotopia nodular & Frontal \\
\hline 56 & Epilepsia com crise tônico-clônica apenas & Generalizada \\
\hline
\end{tabular}


Anexo 2 - Caracterização dos pacientes farmacossensíveis quanto à etiologia

\begin{tabular}{|c|c|c|}
\hline Paciente & Etiologia da epilepsia & Área acometida \\
\hline 1 & Esclerose mesial temporal (EMT) & Temporal \\
\hline 2 & Focal indeterminada & Temporal \\
\hline 3 & Gliose & Frontotemporal \\
\hline 4 & Epilepsia mioclônica juvenil (EMJ) & Generalizada \\
\hline 5 & EMJ & Generalizada \\
\hline 6 & EMT & Temporal \\
\hline 7 & Focal indeterminada & Frontal \\
\hline 8 & Focal indeterminada & Temporal \\
\hline 9 & Epilepsia com crise tônico-clônica apenas & Generalizada \\
\hline 10 & Neurofibromatose & Temporal \\
\hline 11 & Esquizencefalia & Frontal \\
\hline 12 & Focal indeterminada & Temporal \\
\hline 13 & EMJ & Generalizada \\
\hline 14 & EMJ & Generalizada \\
\hline 15 & Displasia cortical & Frontal \\
\hline 16 & EMJ & Generalizada \\
\hline 17 & Gliose & Frontal \\
\hline 18 & DNET & Temporal \\
\hline 19 & Porencefalia & Frontal \\
\hline 20 & Focal indeterminado & Frontal \\
\hline 21 & EMJ & Generalizada \\
\hline 22 & Heterotopia & Frontal \\
\hline 23 & EMJ & Generalizada \\
\hline 24 & EMJ & Generalizada \\
\hline 25 & Focal indeterminada & Temporal bilatera \\
\hline 26 & Epilepsia com crise tônico-clônica apenas & Generalizada \\
\hline 27 & Epilepsia com crise tônico-clônica apenas & Generalizada \\
\hline 28 & Displasia amigdaliana & Temporal \\
\hline 29 & EMJ & Generalizada \\
\hline 30 & EMT & Temporal \\
\hline 31 & Gliose & Frontal \\
\hline 32 & Focal indeterminado & Occipital \\
\hline
\end{tabular}


Anexo 2 - Caracterização dos pacientes farmacossensíveis quanto à etiologia (conclusão)

\begin{tabular}{|c|c|c|}
\hline 33 & EMJ & Generalizada \\
\hline 34 & Focal indeterminada & Temporal \\
\hline 35 & Epilepsia com crise tônico-clônica apenas & Generalizada \\
\hline 36 & Focal indeterminada & Generalizada \\
\hline 37 & EMJ & Generalizada \\
\hline 38 & Gliose & Occipital \\
\hline 39 & EMT & Temporal \\
\hline 40 & Má formação & Occipital \\
\hline 41 & Focal indeterminada & Frontal \\
\hline 42 & EMJ & Generalizada \\
\hline 43 & Neurocisticercose & Multifocal \\
\hline 44 & Focal & Occipital \\
\hline 45 & Ulegiria & Frontal \\
\hline 46 & EMJ & Generalizada \\
\hline 47 & Focal & Indeterminada \\
\hline 48 & Displasia & Frontopolar \\
\hline 49 & Focal indeterminada & Frontal \\
\hline 57 & Gliose por traumatismo crânio encefálico & Multifocal \\
\hline 51 & Gliose & Frontal bilateral \\
\hline 52 & EMJ & Generalizada \\
\hline 53 & EMJ & Generalizada \\
\hline 54 & Gliose & Têmporo-parieta \\
\hline 55 & Focal indeterminada & Indeterminada \\
\hline 56 & EMJ & Generalizada \\
\hline 57 & Gliose & $\begin{array}{l}\text { Fronto-têmporo- } \\
\text { parietal }\end{array}$ \\
\hline 58 & Esquizencefalia & $\begin{array}{c}\text { Frontoparietal } \\
\text { bilateral }\end{array}$ \\
\hline
\end{tabular}

